\title{
Relativistic Kinematics
}

\author{
Raghunath Sahoo* \\ Indian Institute of Technology Indore, India-452020
}

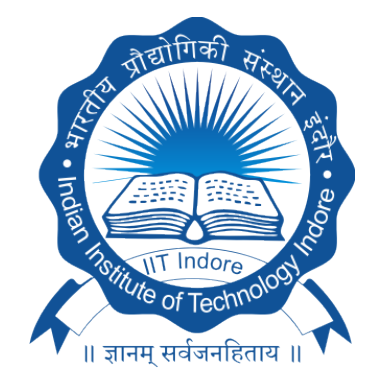

* Email: Raghunath.Sahoo@cern.ch, (Lecture delivered in IX SERC School on Experimental High Energy Physics, IIT Madras, India,12-14 Dec. 2013) 


\section{WHY HIGH ENERGIES?}

In Particle Physics, we deal with elementary constituents of matter. By elementary we mean the particles have no substructure or they are point-like objects. However, the elementariness depends on the spatial resolution of the probe used to investigate the possible structure/sub-structure [1]. The resolution is $\Delta r$ if two points in an object can just be resolved as separate when they are a distance $\Delta r$ apart. Assuming that the probing beams themselves consist of point-like particles like electrons or positrons, the resolution is limited by the de Broglie wavelength of these beam particles, which is given by $\lambda=h / p$, where $p$ is the beam momentum and $h$ is Planck's constant. Hence beams of high momentum have short de Broglie wavelengths and can have high resolution. For example if we need to probe a dimension of 1 Fermi $\left(10^{-15}\right.$ meter) (let's say the inner structure of proton, the charge radius of proton being $\sim 0.840 \mathrm{fm}$ ), we need to use a beam of momentum $1.47 \mathrm{GeV}$ which is given by de Broglie's above expression. Figure 1 shows a schematic picture where a low energy probe fails to probe the inner structure of an object unlike a high energy probe with higher resolution. In addition to the above consideration, Einstein's formula $E=m c^{2}$ helps us to produce particles of higher masses (like the massive gauge bosons, Higgs etc.) in nature's way.

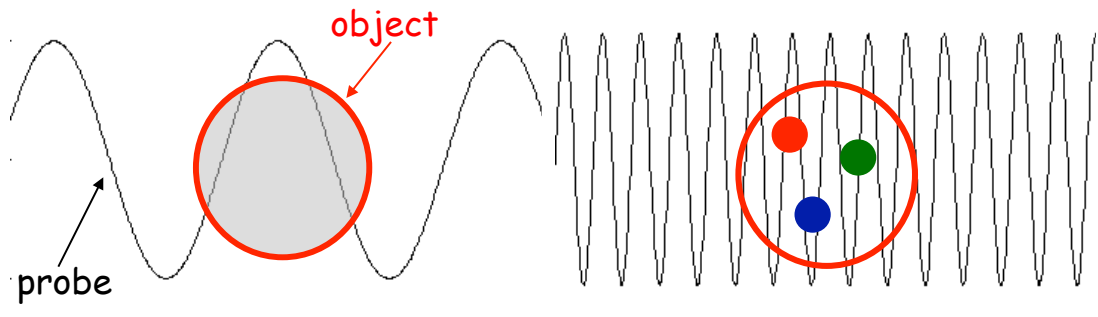

FIG. 1: Left: A low-energy probe probing an object, Right: a high-energy probe able to probe deep inside of the object because of higher resolution.

\section{SPECIAL THEORY OF RELATIVITY \& INVARIANTS}

In particle physics, the particles are treated relativistically, meaning $E \approx p c \gg m c^{2}$ and thus special theory of relativity becomes an mathematical tool in describing the particle kinematics.

- Space and time can not be treated independently as is done in Newtonian mechanics.

- Physical objects that were treated as an independent three component vector and a scalar in non-relativistic physics mix in high-energy phenomena.

- Combined to form a four-component Lorentz vector that transforms like a time and space coordinate.

For their consistent and unified treatment, one relies on Einstein's theory of special relativity (STR), having the following two underlying principles. 
- Invariance of velocity of light: velocity of light always remains as the constant $c$ in any inertial frame.

- Relativity Principle: This requires covariance of the equations, namely the physical law should keep its form invariant in any inertial frame of reference. In mathematical language this amounts to the fact that physical laws have to be expressed in Lorentz tensors.

Note that the principle of relativity applies to Galilei transformation and is valid in Newtonian mechanics as well. But the invariance of the velocity of light necessitates Lorentz transformation in changing from one inertial system to another that are moving relative to each other with constant speed.

\section{A. Lorentz Transformation}

Consider a Lorentz boost in $x$-direction. Here, a particle at $(t, x, y, z$,$) in a coordinate$ frame $L$ is boosted to $\left(t^{\prime}, x^{\prime}, y^{\prime}, z^{\prime}\right)$ with velocity $v$. This statement is equivalent to changing to another coordinate frame $L^{\prime}$ which is moving in the $x$-direction at velocity $-v . L^{\prime}$ is assumed to coincide with $L$ at $t=t^{\prime}=0$. Then the two coordinates are related by the following equations:

$$
\begin{aligned}
& t \rightarrow t^{\prime}=\frac{t+\left(v / c^{2}\right) x}{\sqrt{1-(v / c)^{2}} \Rightarrow x^{0^{\prime}}}=\gamma\left(x^{0}+\beta x\right), \\
& x \rightarrow x^{\prime}=\frac{x+v t}{\sqrt{1-(v / c)^{2}} \Rightarrow x^{1^{\prime}}}=\gamma\left(\beta x^{0}+x\right), \\
& x^{2^{\prime}}= \\
& x^{3^{\prime}}= x^{2}, \\
& x^{3}
\end{aligned}
$$

where, $\beta=v / c, \gamma=\frac{1}{\sqrt{1-\beta^{2}}}$. The above equations can be written in matrix form as:

$$
\left[\begin{array}{l}
x^{0^{\prime}} \\
x^{1^{\prime}} \\
x^{2^{\prime}} \\
x^{3^{\prime}}
\end{array}\right]=\left[\begin{array}{cccc}
\gamma & \beta \gamma & 0 & 0 \\
\beta \gamma & \gamma & 0 & 0 \\
0 & 0 & 1 & 0 \\
0 & 0 & 0 & 1
\end{array}\right] \times\left[\begin{array}{l}
x^{0} \\
x^{1} \\
x^{2} \\
x^{3}
\end{array}\right]
$$

1. The Proper Time $(\tau)$

It is the time an observer feels in the observer's rest frame.

Proper time $d \tau \equiv d t \sqrt{1-\beta^{2}}$ is a Lorentz invariant scalar. Proof: 


$$
\begin{aligned}
d s^{2} & =(c d t)^{2}-d x^{2}-d y^{2}-d z^{2} \\
& =c^{2} d t^{2}\left[1-\frac{d x^{2}}{d t}-\frac{d y^{2}}{d t}-\frac{d z^{2}}{d t}\right] \\
& =c^{2} d t^{2}\left(1-\beta^{2}\right) \\
& =(c d \tau)^{2}
\end{aligned}
$$

is Lorentz invariant by definition.

\section{B. What is need of the variable called "Rapidity"?}

Successive Lorentz boost in the same direction is represented by a single boost, where the transformation velocity is given by

$$
\beta^{\prime \prime}=|v / c|^{\prime \prime}=\frac{\beta+\beta^{\prime}}{1+\beta \beta^{\prime}}
$$

\section{Proof:}

Assume velocity $v^{\prime}$ in frame $L$ is observed as $v^{\prime \prime}$ in frame $L^{\prime \prime}$, where the frame $L^{\prime}$ is travelling in the $x$-direction with $-v$ in frame $L$. The coordinates $\left(t^{\prime}, x^{1^{\prime}}\right)$ are expressed in terms of $\left(t, x^{1}\right)$ using the usual Lorentz transformation equations given by Eqn. 11. Omitting other coordinates for simplicity, one obtains:

$$
\begin{aligned}
x^{0^{\prime}} & =\gamma\left(x^{0}+\beta x^{1}\right) \\
x^{1^{\prime}} & =\gamma\left(\beta x^{0}+x^{1}\right) \\
\beta^{\prime} & =\frac{v^{\prime}}{c}=\frac{d x^{1}}{d x^{0}}
\end{aligned}
$$

then

$$
\begin{aligned}
\beta^{\prime \prime}= & \frac{d x^{1^{\prime}}}{d x^{0^{\prime}}}=\frac{\gamma\left(\beta d x^{0}+d x^{1}\right)}{\gamma\left(d x^{0}+\beta d x^{1}\right)} \\
& \Rightarrow \beta^{\prime \prime}=\frac{\beta+\beta^{\prime}}{1+\beta \beta^{\prime}}
\end{aligned}
$$

The velocity is not an additive quantity. i.e. non-linear in successive transformation. Here comes the need of "Rapidity" to circumvent this drawback, by defining

$\beta=\tanh y$ or $y=\frac{1}{2} \ln \left(\frac{1+\beta}{1-\beta}\right)$. One can show (we will be showing this in subsequent sections) that rapidity is an additive quantity i.e.

$$
y^{\prime \prime}=y+y^{\prime}
$$

Using the rapidity, a Lorentz transformation with finite $\eta$, can be decomposed into $N$ successive transformations with rapidity 
$\Delta y=y / N$

Solving $\beta, \gamma$ in terms of $y$, we have

$\beta=\tanh y, \gamma=\cosh y, \beta \gamma=\sinh y$

Lorentz boost given by Eqn. 1 can be rewritten as

$$
\begin{aligned}
& x^{0^{\prime}}=(\cosh y) x^{0}+(\sinh y) x^{1}, \\
& x^{1^{\prime}}=(\sinh y) x^{0}+(\cosh y) x^{1},
\end{aligned}
$$

Comparing this with rotation in the $x-y$ plane:

$$
\begin{aligned}
& x^{\prime}=x \cos \theta-y \sin \theta, \\
& y^{\prime}=x \sin \theta+y \cos \theta,
\end{aligned}
$$

Eqn. 4 can be obtained from Eqn. 5 by substituting

- $\theta \rightarrow-i y$ (this $y$ is rapidity variable )

- $x \rightarrow i x^{0}$

- $y \rightarrow x^{1}$ (this $y$ is the Cartesian coordinate )

Lorentz boost (in the $x$-direction) is formally a rotation by an angle $(-i y)$ in the $x$ and imaginary time $\left(i x^{0}\right)$ plane.

Experimental Consideration: In high-energy collider experiments, the secondary particles which are produced from the interaction, are boosted in the $z$-direction (along the beam axis). The boosted angular distribution is better expressed as rapidity distribution. At high-energies, each particle has $E \sim p c, p_{I I}=p \cos \theta$, and its rapidity is approximated by so-called pseudo-rapidity:

$$
\eta^{\prime}=\frac{1}{2} \ln \left(\frac{1+\beta_{I I}}{1-\beta_{I I}}\right)=\frac{1}{2} \ln \left(\frac{E+p_{I I} c}{E-p_{I I} c}\right) \sim-\ln \tan \theta / 2 .
$$

This fact is taken into account in designing detectors, which are divided into modules that span the same solid angle in the $\eta-\phi$ (azimuthal angle) plane.

\section{Four Vectors}

Th position-time 4-vector: $x^{\mu}, \mu=0,1,2,3$; with $x^{0}=$ ct, $x^{1}=x, x^{2}=y, x^{3}=z$.

$$
\begin{aligned}
I & \equiv\left(x^{0}\right)^{2}-\left(x^{1}\right)^{2}-\left(x^{2}\right)^{2}-\left(x^{3}\right)^{2} \\
& =\left(x^{0^{\prime}}\right)^{2}-\left(x^{1^{\prime}}\right)^{2}-\left(x^{2^{\prime}}\right)^{2}-\left(x^{3^{\prime}}\right)^{2}
\end{aligned}
$$

$I$ is called the 4-dimensional length element, which is Lorentz Invariant (LI). A quantity having same value in all inertial frames is called an "invariant". This is like $r^{2}=x^{2}+y^{2}+z^{2}$ 
being invariant under spatial rotation. I could be written in the form of a sum:

$$
I=\sum_{\mu=0}^{3} x^{\mu} x^{\mu}
$$

To take care of the negative signs, let's define a "metric" $g_{\mu \nu}$ such that

$$
g=\left[\begin{array}{cccc}
1 & 0 & 0 & 0 \\
0 & -1 & 0 & 0 \\
0 & 0 & -1 & 0 \\
0 & 0 & 0 & -1
\end{array}\right]
$$

Now

$$
I=g_{\mu \nu} x^{\mu} x^{\nu}
$$

Define covariant 4 -vector $x_{\mu}$ (index down):

$$
x_{\mu}=g_{\mu \nu} x^{\nu}
$$

$x^{\mu}$ (index up) is called "contravariant 4 -vector". With the above definitions, now

$$
I=x_{\mu} x^{\mu}=x^{\mu} x_{\mu}
$$

To each contravariant 4 -vector $a^{\mu}$, a covariant 4-vector could be assigned and vice-versa.

$$
\begin{aligned}
& a^{\mu}=g^{\mu \nu} a_{\nu} \\
& a_{\mu}=g_{\mu \nu} a^{\nu}
\end{aligned}
$$

$g^{\mu \nu}$ are the elements in $g^{-1}$. Since $g^{-1}=g, g^{\mu \nu}=g_{\mu \nu}$.

Given any two 4 -vectors, $a^{\mu}$ and $b^{\mu}$,

$$
a^{\mu} b_{\mu}=a_{\mu} b^{\mu}=a^{0} b^{0}-a^{1} b^{1}-a^{2} b^{2}-a^{3} b^{3}
$$

is L.I.. The above operation is called "4-vector scalar product". Remember Einstein's summation convention (repeated Greek indices are to be summed).

$$
\begin{gathered}
a . b \equiv a_{\mu} b^{\mu} \\
=a^{0} b^{0}-\vec{a} \cdot \vec{b} \\
a^{2} \equiv a . a=\left(a^{0}\right)^{2}-\vec{a}^{2}
\end{gathered}
$$

The 1 st term is called "temporal" and the 2nd is called spatial component.

- If $a^{2}>0$ : $a^{\mu}$ is called time-like. Events are in the forward light-cone. They appear later than the origin, $O$. Events in the backward light-cone appear earlier to $O$. Only events in the backward light-cone can influence $O$. And $O$ can have an influence only on the events in the forward cone. 
- If $a^{2}<0$ : $a^{\mu}$ is called space-like. Events are called space-like events and there is no interaction with $O$. This is related to "causality".

- If $a^{2}=0: a^{\mu}$ is called light-like. Connects all those events with the origin which can be reached by a light signal.

This is shown pictorially in Figure 2.

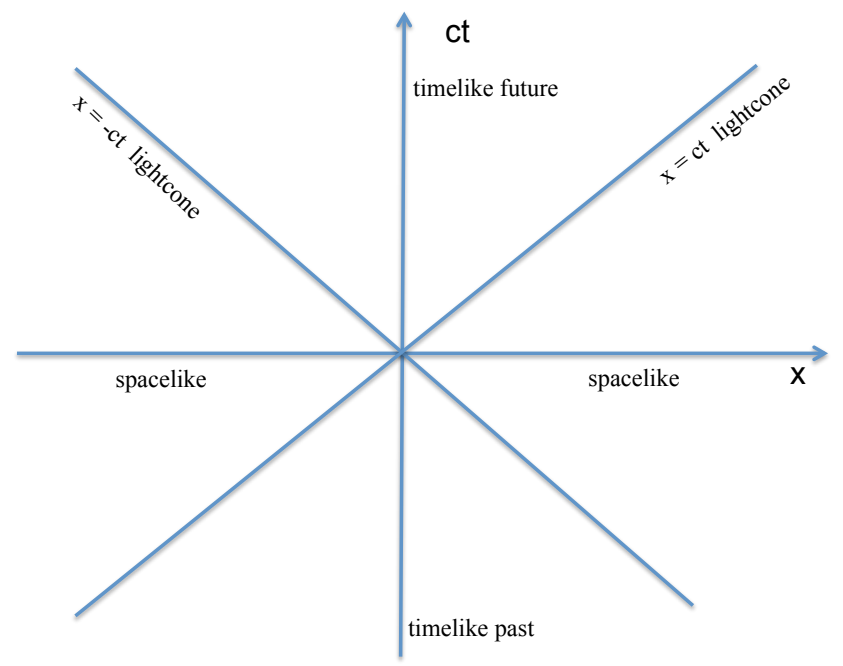

FIG. 2: A schematic of lightcone diagram.

\section{Energy-Momentum Four-Vector}

The velocity of a particle is given by

$$
\vec{v}=\frac{d \vec{x}}{d t}
$$

where $d \vec{x}$ is the distance travelled in the laboratory frame and $d t$ is the time measured in the same frame. Proper velocity of the particle is given by

$$
\vec{\eta}=\frac{d \vec{x}}{d \tau}
$$

where $d \vec{x}$ is the distance travelled in the laboratory frame and $d \tau$ is the proper time. Now

$$
\begin{aligned}
\vec{\eta}=\frac{d \vec{x}}{d \tau} & =\frac{d \vec{x}}{d t} \frac{d t}{d \tau} \\
& =\vec{v} \gamma \\
& \Rightarrow \vec{\eta}=\gamma \vec{v}
\end{aligned}
$$

It is easy to work with the proper velocity, $\vec{\eta}$, as only $d \vec{x}$ transforms under Lorentz transformation. Furthermore,

$$
\eta^{\mu}=\frac{d x^{\mu}}{d \tau}
$$


So

$$
\eta^{0}=\frac{d x^{0}}{d \tau}=\frac{d(c t)}{\frac{1}{\gamma} d t}=\gamma c
$$

Hence

$$
\eta^{\mu}=\gamma\left(c, v_{x}, v_{y}, v_{z}\right)
$$

This is called the proper velocity 4-vector. Remember that the spatial component brings up the negative sign for covariant tensor. Now

$$
\begin{aligned}
\eta^{\mu} \eta_{\mu} & =\gamma^{2}\left(c^{2}-v_{x}^{2}-v_{y}^{2}-v_{z}^{2}\right) \\
& =\gamma^{2} c^{2}\left(1-\frac{v^{2}}{c^{2}}\right) \\
& =\gamma^{2} c^{2}\left(1-\beta^{2}\right) \\
& =c^{2}
\end{aligned}
$$

which is Lorentz Invariant. This also proves that 4-vector scalar product is L.I.

We know momentum = mass $\times$ velocity. And velocity can be "ordinary velocity" or "proper velocity". Classically, both are equal (non-relativistic limit). If $\vec{p}=m \vec{v}$, the conservation of momentum is inconsistent with the principle of relativity. In relativity, momentum is the product of mass and proper velocity.

$$
\begin{gathered}
\vec{p} \equiv m \vec{\eta} \\
p^{\mu}=m \eta^{\mu}
\end{gathered}
$$

The spatial component of $p^{\mu}$ constitutes the (relativistic) momentum 4-vector:

$$
\begin{aligned}
\vec{p}=\gamma m \vec{v} & =\frac{m \vec{v}}{\sqrt{1-v^{2} / c^{2}}} \\
p^{0} & =\gamma m c
\end{aligned}
$$

Relativistic energy, $E$ :

$$
E \equiv \gamma m c^{2}=\frac{m c^{2}}{\sqrt{1-v^{2} / c^{2}}}
$$

Hence,

$$
p^{0}=\frac{E}{c}
$$

and the energy-momentum 4-vector:

$$
p^{\mu}=\left(\frac{E}{c}, p_{x}, p_{y}, p_{z}\right)
$$


Now

$$
\begin{aligned}
p^{\mu} p_{\mu} & =\frac{E^{2}}{c^{2}}-\vec{p}^{2}=m^{2} c^{2}: \text { L.I. } \\
& =\left(m \eta^{\mu}\right)\left(m \eta_{\mu}\right) \\
& =m^{2}\left(\eta^{\mu} \eta_{\mu}\right) \\
& =m^{2} c^{2} \\
\Rightarrow & p^{\mu} p_{\mu}=m^{2} \text { (Natural Units). } \\
& \Rightarrow E^{2}=\vec{p}^{2} c^{2}+m^{2} c^{4}
\end{aligned}
$$

In natural units,

$$
E^{2}=\vec{p}^{2}+m^{2}
$$

- $p^{2}=m^{2}>0$ : Ordinary massive particle

- $p^{2}=m^{2}=0$ : Massless particles like photons, gravitons etc.

- $p^{2}<0$ : Tachyon or virtual particles

- $p^{\mu}=0$ : Vacuum

Remember that the relativistic equations $\vec{p}=\gamma m \vec{v}$ and $E=\gamma m$ do not hold good for massless particles and $m=0$ is allowed only if the particle travels with the speed of light. For massless particles,

$v=c$ and $E=|\vec{p}| c$.

\section{E. The Choice of Units}

We know

$$
\begin{aligned}
& x^{2}=c^{2} t^{2}-x_{1}^{2}-x_{2}^{2}-x_{3}^{2} \\
& p^{2}=m_{0}^{2} c^{2}
\end{aligned}
$$

The velocity of light " $c$ " appears directly in these and many other formulas. Furthermore, de Broglie relation between 4-momentum and wave vector of a particle is

$$
E=\hbar \omega \text { (obtained from Einstein's equation) }
$$

In 4-vector notation,

$$
P=\hbar K
$$

where $P=\left\{\frac{E}{c}, p\right\}, K=\left\{\frac{\omega}{c}, k\right\}$. If we choose a system of unit in which $\hbar=c=1$,

where $\hbar=\frac{h}{2 \pi}=1.055 \times 10^{-34}$ Joule sec: unit of action/angular momentum $\left(M L^{2} / T\right)$. 
$c=2.998 \times 10^{8}$ meter $\sec ^{-1}$ : unit of velocity, the velocity of light in vacuum $(L / T)$.

Now the relativistic formula for energy,

$$
E^{2}=p^{2} c^{2}+m_{0}^{2} c^{4}
$$

in this new system of unit (called natural unit and more popularly used in high-energy (particle) physics) becomes

$$
E^{2}=p^{2}+m_{0}^{2}
$$

We can define system of units completely, if we specify the unit of energy $\left(M L^{2} / T^{2}\right)$. In particle physics, unit of energy is $\mathrm{GeV}\left(1 \mathrm{GeV}=10^{9} \mathrm{eV}\right)$. This choice is motivated by the rest mass of proton $\sim 1 \mathrm{GeV}$. This gives rise to mass $(m)$, momentum $(m c)$, energy $\left(m c^{2}\right)$ in $G e V$. Length $\left(\frac{\hbar}{m c}\right)$ and time $\left(\frac{\hbar}{m c^{2}}\right)$ in $G e V^{-1}$.

Taking the values of $\hbar=c=1$, one obtains,

$$
\begin{aligned}
& 1 \mathrm{sec}=1.52 \times 10^{24} \mathrm{GeV}^{-1} \\
& 1 \text { meter }=5.07 \times 10^{15} \mathrm{GeV}^{-1} \\
& 1 \mathrm{fermi} \equiv 1 \mathrm{fm}=10^{-13} \mathrm{~cm}=10^{-15} \mathrm{~m} \\
& \Rightarrow 1 \mathrm{fm}=5.07 \mathrm{GeV}^{-1} \\
& 1 \mathrm{fm}=3.33 \times 10^{-23} \mathrm{sec} \\
& 197 \mathrm{MeV}=1 \mathrm{fm}^{-1}
\end{aligned}
$$

Note: $1 \mathrm{TeV}=10^{3} \mathrm{GeV}=10^{6} \mathrm{MeV}=10^{9} \mathrm{KeV}=10^{12} \mathrm{eV}$.

The additional advantage of using natural unit in high energy particle physics is that we deal with strong interaction, whose life time $\sim 10^{-23}$ sec, the decay length of particle can be better expressed in terms of fermi.

\section{F. Collider Vs Fixed Target Experiment}

1. For Symmetric Collisions $(A+A)$

Consider the collision of two particles. In LS, the projectile with momentum $\mathbf{p}_{1}$, energy $E_{1}$ and mass $m_{1}$ collides with a particle of mass $m_{2}$ at rest. The 4-momenta of the particles are

$p_{1}=\left(E_{1}, \mathbf{p}_{1}\right), \quad p_{2}=\left(m_{2}, \mathbf{0}\right)$

In CMS, the momenta of both the particles are equal and opposite, the 4-momenta are 


$$
p_{1}^{*}=\left(E_{1}^{*}, \mathbf{p}_{1}^{*}\right), \quad p_{2}^{*}=\left(E_{2}^{*},-\mathbf{p}_{1}^{*}\right)
$$

The total 4-momentum of the system is a conserved quantity in the collision.

In CMS,

$$
\begin{aligned}
p_{\mu} p^{\mu}=\left(p_{1}+p_{2}\right)^{2} & =\left(E_{1}+E_{2}\right)^{2}-\left(\mathbf{p}_{1}+\mathbf{p}_{2}\right)^{2} \\
& =\left(E_{1}+E_{2}\right)^{2} \\
& =E_{c m}^{2} \equiv s
\end{aligned}
$$

$\sqrt{s}$ is the total energy in the CMS which is the invariant mass of the CMS.

In LS,

$$
p_{\mu} p^{\mu}=\left(p_{1}+p_{2}\right)^{2}=m_{1}^{2}+m_{2}^{2}+2 E_{1} m_{2}
$$

Hence

$$
E_{c m}=\sqrt{s}=\sqrt{m_{1}^{2}+m_{2}^{2}+2 E_{\text {proj }} m_{2}}
$$

where $E_{1}=E_{\text {proj }}$, the projectile energy in LS. Hence it is evident here that the CM frame with an invariant mass $\sqrt{s}$ moves in the laboratory in the direction of $\mathbf{p}_{1}$ with a velocity corresponding to:

Lorentz factor,

$$
\begin{aligned}
\gamma_{c m} & =\frac{E_{1}+m_{2}}{\sqrt{s}} \\
\Rightarrow \sqrt{s} & =\frac{E_{l a b}}{\gamma_{c m}},
\end{aligned}
$$

this is because $E=\gamma m$ and

$$
y_{\mathrm{cm}}=\cosh ^{-1} \gamma_{\mathrm{cm}} \text {. }
$$

The center of mass or center of momentum frame (CM/CMS) is at rest and the total momentum is zero. This makes it a suitable choice for solving kinematics problems.

Note: We know that for a collider with head-on collision $\left(\theta=180^{\circ}\right)$

$$
s=E_{c m}^{2}=m_{1}^{2}+m_{2}^{2}+2\left(E_{1} \cdot E_{2}+\left|\mathbf{p}_{1}\right|\left|\mathbf{p}_{2}\right|\right)
$$

For relativistic collisions, $m_{1}, m_{2} \ll E_{1}, E_{2}$

$$
E_{c m}^{2} \simeq 4 E_{1} E_{2}
$$

For two beams crossing at an angle $\theta$,

$$
E_{c m}^{2}=2 E_{1} E_{2}(1+\cos \theta)
$$

The CM energy available in a collider with equal energies $(E)$ for new particle production rises linearly with $E$ i.e.

$$
E_{c m} \simeq 2 E
$$


For a fixed-target experiment the CM energy rises as the square root of the incident energy:

$$
E_{c m} \simeq \sqrt{2 m_{2} E_{1}}
$$

Hence the highest energy available for new particle production is achieved at collider experiments. For example, at SPS fixed-target experiment to achieve a CM energy of 17.3 $\mathrm{AGeV}$ the required incident beam energy is $158 \mathrm{AGeV}$.

Problem: Suppose two identical particles, each with mass $m$ and kinetic energy $T$, collide head-on. What is their relative kinetic energy, $T^{\prime}$ (i.e. K.E. of one in the rest frame of the other). Apply this to an electron-positron collider, where K.E. of electron (positron) is $1 \mathrm{GeV}$. Find the K.E. of electron if positron is at rest (fixed target). Which experiment is preferred, a collider or a fixed-target experiment?

Note: Most of the times the energy of the collision is expressed in terms of nucleonnucleon center of mass energy. In the nucleon-nucleon CM frame, two nuclei approach each other with the same boost factor $\gamma$. The nucleon-nucleon $\mathrm{CM}$ is denoted by $\sqrt{s_{N N}}$ and is related to the total $\mathrm{CM}$ energy by

$$
\sqrt{s}=A \sqrt{s_{N N}}
$$

This is for a symmetric collision with number of nucleons in each nuclei as $A$. The colliding nucleons approach each other with energy $\sqrt{s_{N N}} / 2$ and with equal and opposite momenta. The rapidity of the nucleon-nucleon center of mass is

$y_{N N}=0$ and taking $m_{1}=m_{2}=m_{p}$, the projectile and target nucleons are at equal and opposite rapidities.

$$
y_{\text {proj }}=-y_{\text {target }}=\cosh ^{-1} \frac{\sqrt{s_{N N}}}{2 m_{p}}=y_{\text {beam }} .
$$

Note: Lorentz Factor

$$
\begin{aligned}
\gamma & =\frac{E}{M}=\frac{\sqrt{s}}{2 A m_{p}} \\
& =\frac{A \sqrt{s_{N N}}}{2 A m_{p}}=\frac{\sqrt{s_{N N}}}{2 m_{p}} \\
& =\frac{E_{\text {beam }}^{C M S}}{m_{p}}
\end{aligned}
$$

where $\mathrm{E}$ and $\mathrm{M}$ are Energy and Mass in CMS respectively. Assuming mass of a proton, $m_{p} \sim 1 \mathrm{GeV}$, the Lorentz factor is of the order of beam energy in CMS for a symmetric collision.

\section{For Asymmetric Collisions $(A+B)$}

During the early phase of relativistic nuclear collision research, the projectile mass was limited by accelerator-technical conditions $\left({ }^{38} \mathrm{Ar}\right.$ at the Bevalac, ${ }^{28} \mathrm{Si}$ at the AGS, ${ }^{32} \mathrm{~S}$ at the 


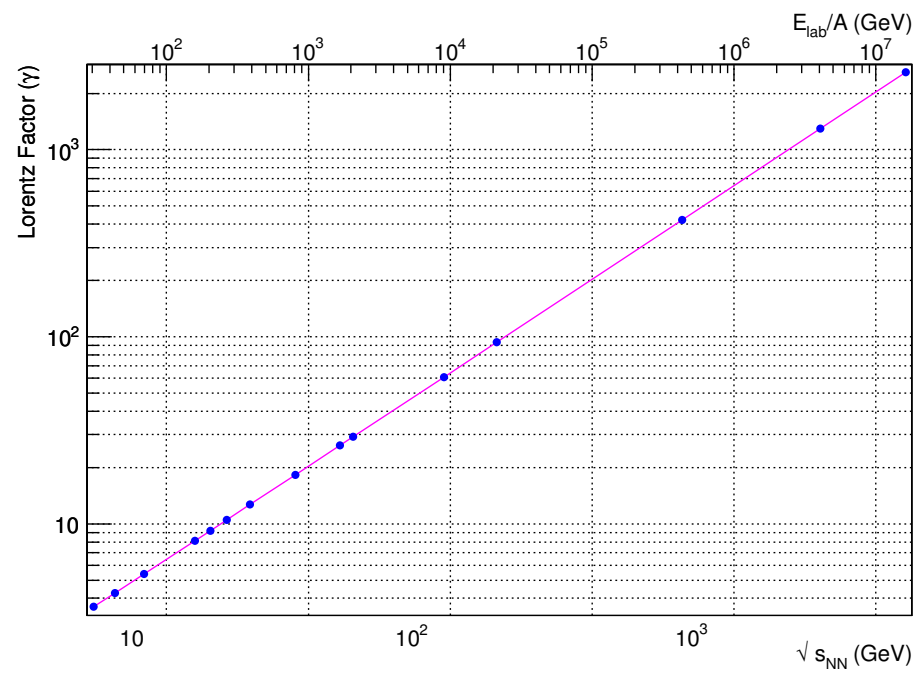

FIG. 3: A plot to demonstrate how the Lorentz Factor increases with collision energy for a symmetric collision. Center of mass system is compared with fixed target experiment.

SPS). Nevertheless, collisions with mass $\approx 200$ nuclear targets were investigated. Analysis of such collisions is faced with the problem of determining an "effective" center of mass frame, to be evaluated from the numbers of projectile and target participant nucleons, respectively. Their ratio - an thus the effective CM rapidity - depends on impact parameter. Moreover, this effective CM frame refers to soft hadron production only, whereas hard processes are still referred to the frame of nucleon-nucleon collisions. The light projectile on heavy target kinematics are described in [17].

The center of mass energy of a collision of two different systems with charge $Z_{1}, Z_{2}$ and atomic numbers $A_{1}, A_{2}$ with $Z=A=1$, for a proton is

$$
\sqrt{s_{N N}} \simeq 2 \sqrt{s_{p p}}+\sqrt{s_{p p}} \sqrt{\frac{Z_{1} Z_{2}}{A_{1} A_{2}}}
$$

where sub-index $N N$ refers to the energy per nucleon inside the colliding nucleus and $\sqrt{s_{p p}}$ is the corresponding energy in $p p$ collisions. The rapidity shift in non-symmetric systems is given by

$$
\Delta y \simeq \frac{1}{2} \ln \left[\frac{Z_{1} A_{2}}{Z_{2} A_{1}}\right]
$$

This is due to the fact that the center-of-mass frame of the $p A$ collision doesn't coincide with the laboratory center-of-mass frame. The rapidity shift in $p+P b$ collision is

$$
\begin{aligned}
\Delta y & \simeq \frac{1}{2} \ln \left[\frac{Z_{1} A_{2}}{Z_{2} A_{1}}\right] \\
& =\frac{1}{2} \ln \left[\frac{82 \times 1}{1 \times 208}\right] \\
& =-0.465
\end{aligned}
$$

Hence $\Delta y= \pm 0.465$ for $p+P b$ collisions, flipping the beams. This rapidity shift need to be taken into account for the comparison with $\mathrm{Pb}+\mathrm{Pb}$ data. 


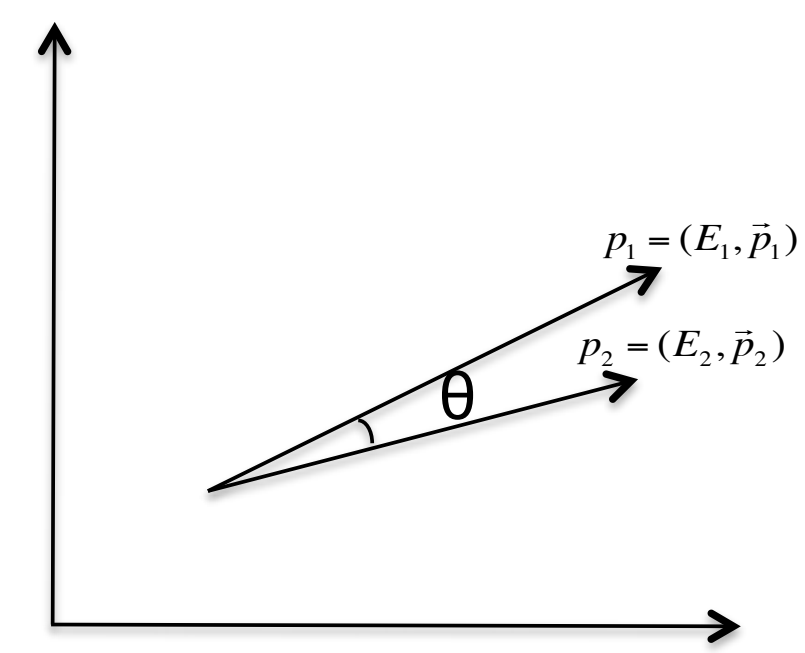

FIG. 4: The Laboratory system: two particle kinematics.

At LHC, the maximum proton beam energy is $7 \mathrm{TeV}$, while the maximum $\mathrm{Pb}$ beam energy is $2.75 \mathrm{TeV}, \sqrt{s}=8.775 \mathrm{TeV}$. The difference in available energy is due to the chargeto-mass ratio, $Z / A$. More is the number of neutrons in the nucleus, difficult it is to accelerate to higher energies. Because of different energies, the two beams will also not have the same rapidity. For the proton beam $y_{p}=9.61$ and for the $\mathrm{Pb}$ beam it is $y_{P b}=8.67$. Thus the center of the collision is shifted away from $y_{c m}=0$ by $\Delta y_{c m}=\left(y_{p}-y_{P b}\right) / 2=0.47$.

\section{G. The Energy and Velocity of the Center of Momentum}

As discussed in Ref. [2], lets consider a Lorentz system- call it the laboratory system (frame), with two particles with masses, $m_{1}$ and $m_{2}$ and four momenta $p_{1}$ and $p_{2}$, respectively as shown in Fig. 4 .

What is the centre of mass energy, $E$ ?

1. It is independent of the Lorentz system, where $p_{1}$ and $p_{2}$ are defined.

2. It must be possible to have an answer in terms of the three invariants, namely $p_{1}^{2}=m_{1}^{2}$ and $p_{2}^{2}=m_{2}^{2}$ and $\left[p_{1} p_{2}\right.$ or $\left(p_{1}+p_{2}\right)^{2}$ or $\left.\left(p_{1}-p_{2}\right)^{2}\right]$.

The answer would be trivial in the center of momentum (mass) frame itself. We use asterisk $(*)$ in the CM frame to distinguish that from the laboratory frame.

In CM frame,

$$
\begin{gathered}
\left({\overrightarrow{p_{1}}}^{*}+{\overrightarrow{p_{2}}}^{*}\right)=0 \\
\Longrightarrow p_{1}+p_{2}=\left(E_{1}^{*}+E_{2}^{*}, \overrightarrow{0}\right) \\
\text { and } E^{*}=E_{1}^{*}+E_{2}^{*}
\end{gathered}
$$


Hence,

$$
\begin{aligned}
\left(E^{*}\right)^{2} & =\left(E_{1}^{*}+E_{2}^{*}\right)^{2} \\
& =\left(p_{1}^{*}+p_{2}^{*}\right)^{2} \\
& =\left(p_{1}+p_{2}\right)^{2} .
\end{aligned}
$$

This is because $\left(p_{1}+p_{2}\right)^{2}$ is invariant.

Let us define the total mass $M$ of the system as the square of the total 4-momentum as:

$$
M^{2}=\left(p_{1}+p_{2}\right)^{2}=P^{2}=\left(E^{*}\right)^{2}=\left(E_{1}+E_{2}\right)^{2}-\left(p_{1}+p_{2}\right)^{2}=\text { Invariant }
$$

Kinematically, for the given system, the two particles $p_{1}$ and $p_{2}$ are equivalent to one single particle with 4-momentum $P$ and mass $M=E_{\mathrm{CM}}$. Generalizing this, one can consider these individual particles to represent a system of particles.

Further, we know

$\vec{p}=m \vec{v} \gamma$, and $\mathrm{E}=\mathrm{m} \gamma$, where $\gamma=\frac{1}{\sqrt{1-\beta^{2}}}$.

Hence, the 4-momentum of the two-particle system is given by:

$\vec{P}=M \vec{\beta} \gamma$

$E=M \gamma$.

Using the above, one obtains:

$$
\begin{aligned}
\beta_{\mathrm{CM}} & =\frac{\vec{P}}{E} \\
& =\frac{\left(\overrightarrow{p_{1}}+\overrightarrow{p_{2}}\right)}{\left(E_{1}+E_{2}\right)},
\end{aligned}
$$

is the velocity of the CM seen from the laboratory system.

$$
\begin{aligned}
\gamma_{\mathrm{CM}} & =\frac{1}{\sqrt{1-\beta^{2}}} \\
& =\frac{E}{M} \\
& =\frac{E_{1}+E_{2}}{\sqrt{\left(E_{1}+E_{2}\right)^{2}-\left(\overrightarrow{p_{1}}+\overrightarrow{p_{2}}\right)^{2}}} \\
& =\frac{E_{1}+E_{2}}{E_{\mathrm{CM}}},
\end{aligned}
$$

is the Lorentz factor or the Lorentz boost of the CM. In general, the Lorentz factor of the CM is the ratio of the sum of the energies of the particles in the laboratory system and the energy of the CM. 
H. The Energy, Momentum and Velocity of one particle as seen from the rest system of another one

Lets assume that we sit on particle 1, which is in motion. What will be the energy of particle 2, for us?

The answer to this question must always be the same, irrespective of the Lorentz system we start with. It is thus expressible by the invariants discussed in the preceding subsection (point-2), where the last two variables are the well-known Mandelstam's $s$ and $t$ variables.

Let $E_{21}$ : is the energy of particle 2 , if we look at it sitting on particle 1 , which then appears to be at rest for us.

$E_{21}=E_{2}$, in the system where $\overrightarrow{p_{1}}=0$. One needs to write $E_{21}$ in terms of the invariants available in this problem.

Now, $p_{1} p_{2}=E_{1} E_{2}-\overrightarrow{p_{1}} \cdot \overrightarrow{p_{2}}=m_{1} E_{2}\left(\right.$ since $\left.\overrightarrow{p_{1}}=0\right)$.

Hence, $E_{21}=E_{2}=\frac{p_{1} p_{2}}{m_{1}}$.

Note that the RHS is already in an invariant form.

$\left|\vec{p}_{21}\right|^{2}=E_{21}^{2}-m_{2}^{2}=\frac{\left(p_{1} p_{2}\right)^{2}-m_{1}^{2} m_{2}^{2}}{m_{1}^{2}}$.

If $p_{1}$ and $p_{2}$ are the momentum 4-vectors of any two particles in any Lorentz system, then

$$
\begin{array}{r}
E_{21}=\frac{p_{1} p_{2}}{m_{1}} \\
\left|\vec{p}_{21}\right|^{2}=\frac{\left(p_{1} p_{2}\right)^{2}-m_{1}^{2} m_{2}^{2}}{m_{1}^{2}} \quad\left(p_{1} p_{2} \equiv E_{1} E_{2}-\vec{p}_{1} \vec{p}_{2}\right) \\
v_{21}^{2}=\frac{\left|\vec{p}_{21}\right|^{2}}{E_{21}^{2}}=\frac{\left(p_{1} p_{2}\right)^{2}-m_{1}^{2} m_{2}^{2}}{\left(p_{1} p_{2}\right)^{2}}
\end{array}
$$

The above three equations give the energy, momentum and velocity of particle 2 , as seen from particle 1 . The velocity $v_{21}$ is the relative velocity, which is symmetric in 1 and 2 . Note here that all these expressions are invariant and can be evaluated in any Lorentz system.

\section{The Energy, Momentum, and Velocity of a Particle as seen from the CM System}

This problem is now like all the above quantities are as seen from a fictitious particle $M$, called the "center-of-momentum-particle", whose 4-momentum is

$$
P=p_{1}+p_{2}
$$

We need to apply formulae (Eq. 62) with $p_{1}$ replaced by $P$ and $p_{2}$ by the 4 -momentum 
of that particle whose energy, momentum and velocity, we like to determine. From Eq. 62 ,

$$
\begin{array}{r}
E_{1}^{*}=\frac{P p_{1}}{M} \\
\left|\vec{p}_{1}^{*}\right|^{2}=\frac{\left(P p_{1}\right)^{2}-M^{2} m_{1}^{2}}{M^{2}} \\
v_{1}^{* 2}=\frac{\left(P p_{1}\right)^{2}-M^{2} m_{1}^{2}}{\left(P p_{1}\right)^{2}}
\end{array}
$$

This is by using Eq. 63 and by using

$$
\begin{aligned}
p_{1} p_{2} & =\frac{1}{2}\left[\left(p_{1}+p_{2}\right)^{2}-p_{1}^{2}-p_{2}^{2}\right] \\
& =\frac{1}{2}\left(M^{2}-m_{1}^{2}-m_{2}^{2}\right),
\end{aligned}
$$

one obtains

$$
\begin{gathered}
E_{1}^{*}=\frac{M^{2}+\left(m_{1}^{2}-m_{2}^{2}\right)}{2 M} \\
E_{2}^{*}=\frac{M^{2}-\left(m_{1}^{2}-m_{2}^{2}\right)}{2 M} \\
E_{1}^{*}+E_{2}^{*}=M \\
\left|\vec{p}^{*}\right|^{2}=\left|\vec{p}_{1}^{*}\right|^{2}=\left|\vec{p}_{2}^{*}\right|^{2} \\
=\frac{M^{4}-2 M^{2}\left(m_{1}^{2}+m_{2}^{2}\right)+\left(m_{1}^{2}-m_{2}^{2}\right)}{4 M^{2}} \\
=\frac{\left[M^{2}-\left(m_{1}+m_{2}\right)^{2}\right]\left[M^{2}-\left(m_{1}-m_{2}\right)^{2}\right]}{4 M^{2}} \\
v_{1}^{* 2}=\left(\frac{\left|\vec{p}^{*}\right|}{E_{1}^{*}}\right)^{2} .
\end{gathered}
$$

Here, $E_{1}^{*}, v_{1}{ }^{*}$ are energy and velocity of particle 1 , as seen from their common CM system and $M^{2}=P^{2}=\left(p_{1}+p_{2}\right)^{2}$ is the square of the total mass. The above equations give the energy, momenta and the velocities of two particles $m_{1}$ and $m_{2}$ for which

$$
M \rightarrow m_{1}+m_{2}
$$

\section{J. Description of Nucleus-Nucleus Collisions in terms of Light-Cone Variables}

In relativistic nucleus-nucleus collisions, it is convenient to use kinematic variables which take simple forms under Lorentz transformations for the change of frame of reference. A few of them are the light cone variables $x_{+}$and $x_{-}$, the rapidity and pseudorapidity variables, $y$ and $\eta$. A particle is characterized by its 4 -momentum, $p_{\mu}=(E, \mathbf{p})$. In fixed target and collider experiments where the beam(s) define reference frames, boosted along their 


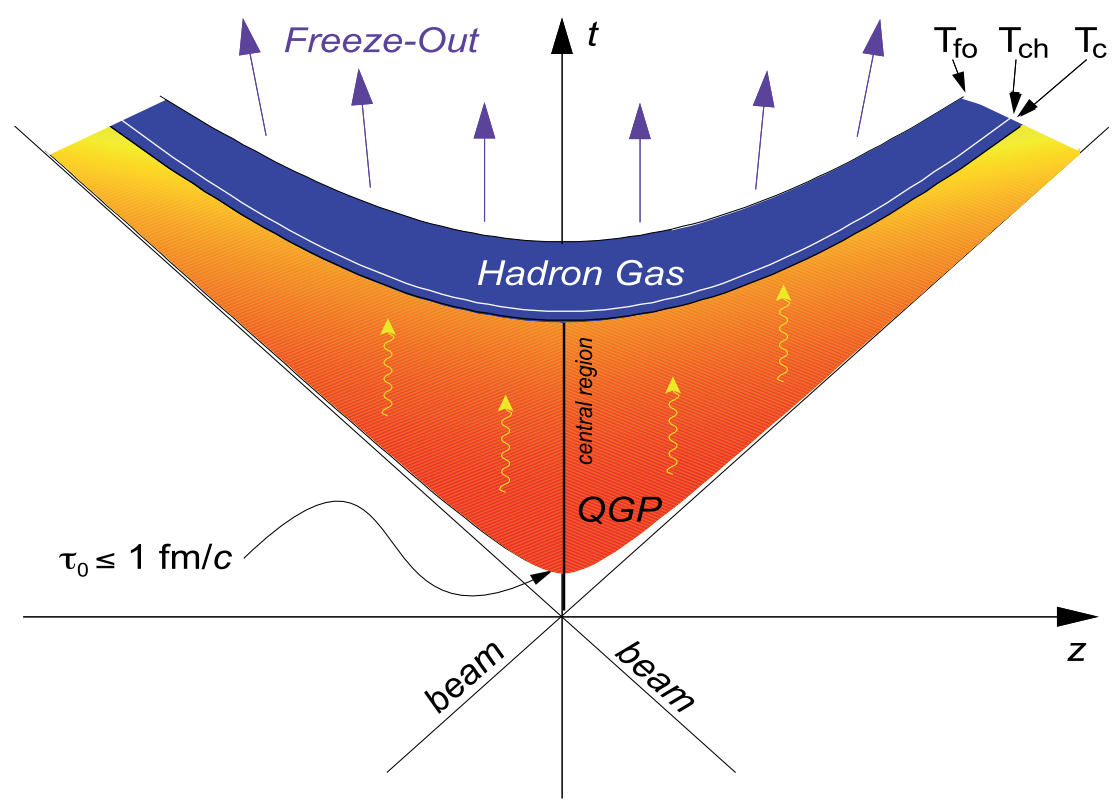

FIG. 5: Description of heavy-ion collisions in one space $(z)$ and one time $(t)$ dimension.

direction, it is important to express the 4-momentum in terms of more practical kinematic variables.

Figure 5 shows the collision of two Lorentz contracted nuclei approaching each other with velocities nearly equal to velocity of light. The vertical axis represents the time direction with the lower half representing time before the collision and the upper half, time after the collision. The horizontal axis represents the spatial direction. Both the nuclei collide at $(t, z)=(0,0)$ and then the created fireball expands in time going through various processes till the created particles freeze-out and reach the detectors. The lines where $t^{2}-z^{2}=0$ (note that $\sqrt{t^{2}-z^{2}} \equiv \tau, \tau$ being the proper time of the particle) along the path of the colliding nuclei define the light cone. The upper part of the light-cone, where $t^{2}-z^{2}>0$, is the time-like region. In nucleus-nucleus collisions, particle production occurs in the upper half of the $(t, z)$-plane within the light-cone. The region outside the light cone for which $t^{2}-z^{2}<0$ is called space-like region. The space-time rapidity is defined as

$$
\eta_{s}=\frac{1}{2} \ln \left(\frac{t+z}{t-z}\right)
$$

It could be seen that $\eta_{s}$ is not defined in the space-like region. It takes the value of positive and negative infinity along the beam directions for which $t= \pm z$ respectively. A particle is "light-like" along the beam direction. Inside the light-cone which is time-like, $\eta_{s}$ is properly defined.

For a particle with 4-momentum $p\left(p_{0}, \mathbf{p}_{\mathbf{T}}, p_{z}\right)$, the light-cone momenta are defined by

$$
\begin{aligned}
& p_{+}=p_{0}+p_{z} \\
& p_{-}=p_{0}-p_{z}
\end{aligned}
$$

$p_{+}$is called "forward light-cone momentum" and $p_{-}$is called "backward light-cone momentum".

For a particle travelling along the beam direction, has higher value of forward light-cone 
momentum and travelling opposite to the beam direction has lower value of forward lightcone momentum. The advantages of using light-cone variables to study particle production are the following.

1. The forward light-cone momentum of any particle in one frame is related to the forward light-cone momentum of the same particle in another boosted Lorentz frame by a constant factor.

2. Hence, if a daughter particle $c$ is fragmenting from a parent particle $b$, then the ratio of the forward light-cone momentum of $c$ relative to that of $b$ is independent of the Lorentz frame.

Define

$$
\begin{aligned}
x_{+} & =\frac{p_{0}^{c}+p_{z}^{c}}{p_{0}^{b}+p_{z}^{b}} \\
& =\frac{c_{+}}{b_{+}} .
\end{aligned}
$$

The forward light-cone variable $x_{+}$is always positive because $c_{+}$can't be greater than $b_{+}$. Hence the upper limit of $x_{+}$is $1 . x_{+}$is Lorentz invariant.

3. The Lorentz invariance of $x_{+}$provides a tool to measure the momentum of any particle in the scale of the momentum of any reference particle.

\section{K. Pictorial Representation of Detector System}

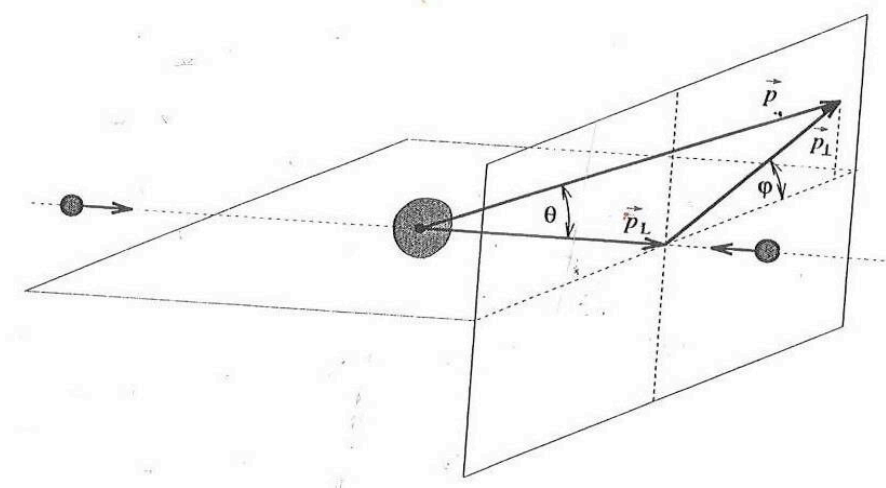

FIG. 6: A schematic decomposition of particle momentum $\vec{p}$ (in CM frame) into parallel and longitudinal components. Note the angle of inclination $\theta$ of $\vec{p}$ and the azimuthal angle $\phi$ of $p_{\perp}$ [3].

In an collider experimental environment, a particle is emitted from the collision point making a polar angle $\theta$ with the collision point. When the momenta of the particles are determined by a tracking detector, the (pseudo)-rapidity and azimuthal angles are given by $y=\tanh ^{-1} v_{z}=\tanh ^{-1} \frac{p_{z}}{E}=\tanh ^{-1} \frac{p_{z}}{\sqrt{p_{x}^{2}+p_{y}^{2}+p_{z}^{2}+m_{0}^{2}}}$

$$
\text { and } \phi=\tan ^{-1} \frac{p_{y}}{p_{x}} \text {. }
$$


The polar angle $\theta$ is given by

$\theta=\cos ^{-1} \frac{p_{z}}{|\vec{p}|}=\tan ^{-1} \frac{\left|\overrightarrow{p_{T}}\right|}{p_{z}}$.

Pictorially, these are shown in the Figure 6. A detector plane is spanned by $(\eta, \phi)$, with $\eta$ decreasing while going away from the beam axis in annular rings and $\phi$ is scanned making an angle with the beam axis and increasing it anti-clock-wise, as is shown in the picture.

\section{The Rapidity Variable}

One of the important tasks in hand is to introduce key kinematic variables that relate particle momentum to the dynamics that is occurring in heavy-ion collisions. It is essentially the convenience of working in center-of-momentum system, we need to introduce the observable rapidity. To do so, let's proceed as follows: The co-ordinates along the beam line (conventionally along the $z$-axis) is called longitudinal and perpendicular to it is called transverse $(x-y)$. The 3-momentum can be decomposed into the longitudinal $\left(p_{z}\right)$ and the transverse $\left(\mathbf{p}_{T}\right), \mathbf{p}_{T}$ being a vector quantity which is invariant under a Lorentz boost along the longitudinal direction. The variable rapidity " $y$ " is defined by

$$
\begin{aligned}
y= & \frac{1}{2} \ln \left(\frac{E+p_{z}}{E-p_{z}}\right) \\
& =\ln \left(\frac{E+p_{z}}{m_{T}}\right)
\end{aligned}
$$

It is a dimensionless quantity related to the ratio of forward light-cone to backward lightcone momentum. The rapidity changes by an additive constant under longitudinal Lorentz boosts.

For a free particle which is on the mass shell (for which $E^{2}=p^{2}+m^{2}$ ), the 4-momentum has only three degrees of freedom and can be represented by $\left(y, \mathbf{p}_{T}\right)$. $\left(E, \mathbf{p}_{T}\right)$ could be expressed in terms of $\left(y, \mathbf{p}_{T}\right)$ as

$$
\begin{aligned}
E & =m_{T} \cosh y \\
p_{z} & =m_{T} \sinh y
\end{aligned}
$$

$m_{T}$ being the transverse mass which is defined as

$$
m_{T}^{2}=m^{2}+\mathbf{p}_{T}^{2}
$$

The advantage of rapidity variable is that the shape of the rapidity distribution remains unchanged under a longitudinal Lorentz boost. When we go from CMS to LS, the rapidity distribution is the same, with the $y$-scale shifted by an amount equal to $y_{\mathrm{cm}}$. This is shown below.

\section{Rapidity of Center of Mass in the Laboratory System}

The total energy in the CMS system is $E_{c m}=\sqrt{s}$. The energy and momentum of the CMS in the LS are $\gamma_{c m} \sqrt{s}$ and $\beta_{c m} \gamma_{c m} \sqrt{s}$ respectively. The rapidity of the CMS in the LS 
is

$$
\begin{aligned}
y_{c m} & =\frac{1}{2} \ln \left[\frac{\gamma_{c m} \sqrt{s}+\beta_{c m} \gamma_{c m} \sqrt{s}}{\gamma_{c m} \sqrt{s}-\beta_{c m} \gamma_{c m} \sqrt{s}}\right] \\
& =\frac{1}{2} \ln \left[\frac{1+\beta_{c m}}{1-\beta_{c m}}\right]
\end{aligned}
$$

It is a constant for a particular Lorentz transformation.

\section{Relationship between Rapidity of a particle in $L S$ and rapidity in $C M S$}

The rapidities of a particle in the LS and CMS of the collision are respectively, $y=$ $\frac{1}{2} \ln \left(\frac{E+p_{z}}{E-p_{z}}\right)$ and $y^{*}=\frac{1}{2} \ln \left(\frac{E^{*}+p_{z}^{*}}{E^{*}-p_{z}^{*}}\right)$. For a particle travelling in longitudinal direction, the Lorentz transformation of its energy and momentum components give

$$
\left[\begin{array}{c}
E^{*} \\
P_{L}^{*}
\end{array}\right]=\left[\begin{array}{cc}
\gamma & -\gamma \beta \\
-\gamma \beta & \gamma
\end{array}\right] \cdot\left[\begin{array}{c}
E \\
P_{L}
\end{array}\right], P_{T}^{*}=P_{T}
$$

where $P_{L}$ and $P_{T}$ are the longitudinal and transverse components of $\vec{P}$, which are parallel and perpendicular to $\beta$, respectively. Hence, the inverse Lorentz transformations on $E$ and $p_{z}$ give

$$
\begin{aligned}
y & =\frac{1}{2} \ln \left[\frac{\gamma\left(E^{*}+\beta p_{z}^{*}\right)+\gamma\left(\beta E^{*}+p_{z}^{*}\right)}{\gamma\left(E^{*}+\beta p_{z}^{*}\right)-\gamma\left(\beta E^{*}+p_{z}^{*}\right)}\right] \\
& =\frac{1}{2} \ln \left[\frac{E^{*}+p_{z}^{*}}{E^{*}-p_{z}^{*}}\right]+\frac{1}{2} \ln \left[\frac{1+\beta}{1-\beta}\right] \\
\Rightarrow y & =y^{*}+y_{c m} .
\end{aligned}
$$

Hence the rapidity of a particle in the laboratory system is equal to the sum of the rapidity of the particle in the center of mass system and the rapidity of the center of mass in the laboratory system. It can also be state that the rapidity of a particle in a moving (boosted) frame is equal to the rapidity in its own rest frame minus the rapidity of the moving frame. In the non-relativistic limit, this is like the subtraction of velocity of the moving frame. However, this is not surprising because, non-relativistically, the rapidity $y$ is equal to longitudinal velocity $\beta$. Rapidity is a relativistic measure of the velocity. This simple property of the rapidity variable under Lorentz transformation makes it a suitable choice to describe the dynamics of relativistic particles. The simple shape invariance nature of rapidity spectra brings its importance in the analysis of particle production in nuclear collisions. For instance, in fixed-target experiments, we can study particle spectra using $y$ as a variable without making an explicit transformation to the CM frame of reference and from the rapidity spectra, we deduce the point of symmetry corresponding to the CM rapidity. In symmetric collisions with fixed targets, the CM frame is located in the middle between the rapidities of the projectile and target i.e. $y_{c m}=y_{\text {proj }} / 2$. In this case, the particle rapidity spectrum must be symmetric around $y_{\mathrm{cm}}$. This allows for complementing measured particle spectra: if these are available for, e.g. $y \geq y_{c m}$, a reflection at the symmetry point $y_{c m}$ gives us the part of the spectrum with $y \leq y_{c m}$, for which an experimental measurement is absent. 


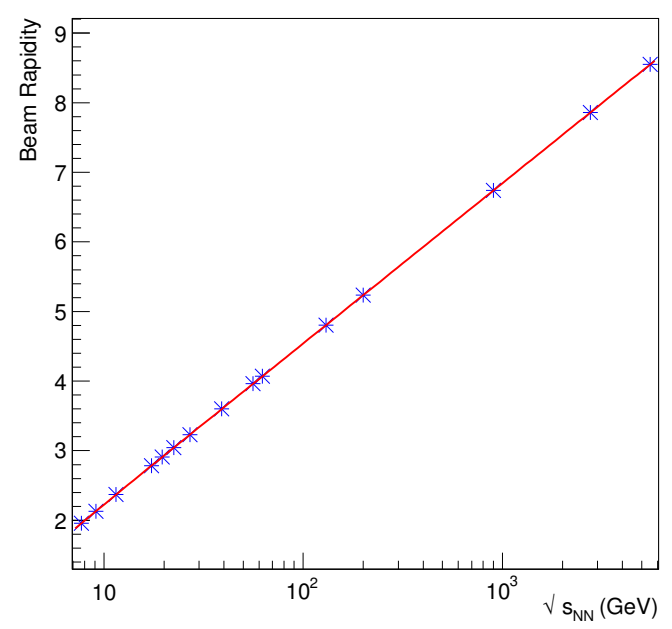

FIG. 7: A plot of beam rapidity as a function of center of mass energy.

\section{Relationship between Rapidity and Velocity}

Consider a particle travelling in $z$-direction with a longitudinal velocity $\beta$. The energy $E$ and the longitudinal momentum $p_{z}$ of the particle are

$$
\begin{aligned}
E & =\gamma m \\
p_{z} & =\gamma \beta m \\
p_{z} & =\beta E
\end{aligned}
$$

where $m$ is the rest mass of the particle. Hence the rapidity of the particle travelling in $z$-direction with velocity $\beta$ is

$$
\begin{aligned}
y_{\beta} & =\frac{1}{2} \ln \left[\frac{E+p_{z}}{E-p_{z}}\right]=\frac{1}{2} \ln \left[\frac{\gamma m+\gamma \beta m}{\gamma m-\gamma \beta m}\right] \\
& =\frac{1}{2} \ln \left[\frac{1+\beta}{1-\beta}\right]
\end{aligned}
$$

Note here that $y_{\beta}$ is independent of particle mass. A particle is said to be relativistic in nature if $\gamma>>1, \beta \approx 1$ and $E \approx p$. In other words, the energy of the particle is much higher than its rest mass i.e. $E>>m_{0}$ and hence the energy and total momentum of the particle are comparable. In the non-relativistic limit when $\beta$ is small, expanding $y_{\beta}$ in terms of $\beta$ leads to

$$
y_{\beta}=\beta+O\left(\beta^{3}\right)
$$

Thus the rapidity of the particle is the relativistic realization of its velocity.

\section{Beam Rapidity}

We know, $E=m_{T} \cosh y, p_{z}=m_{T} \sinh y$ and $m_{T}^{2}=m^{2}+\mathbf{p}_{T}^{2}$. 
For the beam particles, $p_{T}=0$.

Hence, $E=m_{b} \cosh y_{b}$ and $p_{z}=m_{b} \sinh y_{b}$, where $m_{b}$ and $y_{b}$ are the rest mass and rapidity of the beam particles.

$$
y_{b}=\cosh ^{-1}\left(E / m_{b}\right)
$$

Note that Eq. 89 is used for fixed target experiment with $E$ as the energy of individual beam particles, neglecting the rest mass. For example, for SPS beam energy of $158 \mathrm{AGeV}$, $E=158 \mathrm{GeV}$. This leads to $y_{b}=5.82$ in fixed target framework. Now when we convert Eq. 89 for a collider system, we get

$$
\begin{aligned}
y_{b} & =\cosh ^{-1}\left(E / m_{b}\right) \\
& =\cosh ^{-1}\left[\frac{\sqrt{s_{N N}}}{2 m_{p}}\right] \\
& \Rightarrow y_{b}=\ln \left(\sqrt{s_{N N}} / m_{p}\right)
\end{aligned}
$$

Now, for the above SPS beam energy of $158 \mathrm{AGeV}$, the equivalent center of mass energy per nucleon is, $\sqrt{s_{\mathrm{NN}}}=17.3 \mathrm{GeV}$. By using Eq. 91, we get $y_{b}=2.91$. This makes sense to us, as in a collider experiment both the beams come in opposite directions with equal beam rapidities.

Further,

$$
y_{b}=\sinh ^{-1}\left(p_{z} / m_{b}\right)
$$

In terms of the beam velocity, beam rapidity can be expressed as

$$
y_{b}=\tanh ^{-1}\left(p_{z} / E\right)=\tanh ^{-1} \beta
$$

Here $m_{p}$ is the mass of a proton. Note that the beam energy $E=\sqrt{s_{N N}} / 2$ for a symmetric collider.

Furthermore, it could be shown that: $y_{b}=\mp \ln \left(\sqrt{s_{N N}} / m_{p}\right)=\mp y_{\max }$

Example For the nucleon-nucleon center of mass energy $\sqrt{s_{N N}}=9.1 \mathrm{GeV}$, the beam rapidity $y_{b}=\cosh ^{-1}\left(\frac{9.1}{2 \times 0.938}\right)=2.26$

For $\mathrm{p}+\mathrm{p}$ collisions with lab momentum $100 \mathrm{GeV} / \mathrm{c}$,

$y_{b}=\sinh ^{-1}\left(\frac{p_{z}}{m_{b}}\right)=\sinh ^{-1}\left(\frac{100}{0.938}\right)=5.36$

and for $\mathrm{Pb}+\mathrm{Pb}$ collisions at SPS with lab energy $158 \mathrm{AGeV}, y_{b}=2.91$.

TABLE I: Table of collision energy, $\sqrt{s_{N N}}$ Vs the beam rapidity, $y_{b}$.

\begin{tabular}{|c|c|c|c|c|c|c|c|c|c|c|c|c|c|c|c|c|}
\hline$\sqrt{s_{N N}}(\mathrm{GeV})$ & 7.7 & 9.1 & 11.5 & 17.3 & 19.6 & 27.0 & 39.0 & 62.4 & 130 & 200 & 900 & 2360 & 2760 & 5520 & 7000 & 14000 \\
\hline$y_{b}$ & 2.10 & 2.27 & 2.50 & 2.91 & 3.03 & 3.35 & 3.72 & 4.19 & 4.93 & 5.36 & 6.86 & 7.83 & 7.98 & 8.68 & 8.91 & 9.61 \\
\hline & \multicolumn{10}{|c|}{} \\
\hline
\end{tabular}




\section{Rapidity of the CMS in terms of Projectile and Target Rapidities}

Let us consider the beam particle " $b$ " and the target particle " $a$ ".

$b_{z}=m_{T} \sinh y_{b}=m_{b} \sinh y_{b}$. This is because $p_{T}$ of beam particles is zero. Hence

$$
y_{b}=\sinh ^{-1}\left(b_{z} / m_{b}\right) .
$$

The energy of the beam particle in the laboratory frame is

$b_{0}=m_{T} \cosh y_{b}=m_{b} \cosh y_{b}$.

Assuming target particle $a$ has longitudinal momentum $a_{z}$, its rapidity in the laboratory frame is given by

$$
y_{a}=\sinh ^{-1}\left(a_{z} / m_{a}\right)
$$

and its energy

$$
a_{0}=m_{a} \cosh y_{a} .
$$

The CMS is obtained by boosting the LS by a velocity of the center-of-mass frame $\beta_{c m}$ such that the longitudinal momentum of the beam particle $b_{z}^{*}$ and of the target particle $a_{z}^{*}$ are equal and opposite. Hence $\beta_{c m}$ satisfies the condition,

$$
\begin{gathered}
a_{z}^{*}=\gamma_{c m}\left(a_{z}-\beta_{c m} a_{0}\right)=-b_{z}^{*}=-\gamma_{c m}\left(b_{z}-\beta_{c m} b_{0}\right), \text { where } \gamma_{c m}=\frac{1}{\sqrt{1-\beta_{c m}^{2}}} . \text { Hence, } \\
\beta_{c m}=\frac{a_{z}+b_{z}}{a_{0}+b_{0}} .
\end{gathered}
$$

We know the rapidity of the center of mass is

$$
y_{c m}=\frac{1}{2} \ln \left[\frac{1+\beta_{c m}}{1-\beta_{c m}}\right]
$$

Using equations 97 and 98 , we get

$$
y_{c m}=\frac{1}{2} \ln \left[\frac{a_{0}+a_{z}+b_{0}+b_{z}}{a_{0}-a_{z}+b_{0}-b_{z}}\right] .
$$

Writing energies and momenta in terms of rapidity variables in the LS,

$$
\begin{aligned}
y_{c m} & =\frac{1}{2} \ln \left[\frac{m_{a} \cosh y_{a}+m_{a} \sinh y_{a}+m_{b} \cosh y_{b}+m_{b} \sinh y_{b}}{m_{a} \cosh y_{a}-m_{a} \sinh y_{a}+m_{b} \cosh y_{b}-m_{b} \sinh y_{b}}\right] \\
& =\frac{1}{2}\left(y_{a}+y_{b}\right)+\frac{1}{2} \ln \left[\frac{m_{a} e^{y_{a}}+m_{b} e^{y_{b}}}{m_{a} e^{y_{b}}+m_{b} e^{y_{a}}}\right]
\end{aligned}
$$

For a symmetric collision (for $m_{a}=m_{b}$ ),

$$
y_{c m}=\frac{1}{2}\left(y_{a}+y_{b}\right)
$$

Rapidities of $a$ and $b$ in the CMS are

$$
\begin{gathered}
y_{a}^{*}=y_{a}-y_{c m}=-\frac{1}{2}\left(y_{b}-y_{a}\right) \\
y_{b}^{*}=y_{b}-y_{c m}=\frac{1}{2}\left(y_{b}-y_{a}\right) .
\end{gathered}
$$

Given the incident energy, the rapidity of projectile particles and the rapidity of the target particles can thus be determined. The greater is the incident energy, the greater is the separation between the projectile and target rapidity. 
Central Rapidity The region of rapidity mid-way between the projectile and target rapidities is called central rapidity.

Example In $\mathrm{p}+\mathrm{p}$ collisions at a laboratory momentum of $100 \mathrm{GeV} / \mathrm{c}$, beam rapidity $y_{b}=$ $\overline{5.36, \text { target }}$ rapidity $y_{a}=0$ and the central rapidity $\approx 2.7$.

\section{Mid-rapidity in Fixed target and Collider Experiments}

In fixed-target experiments (LS), $y_{\text {target }}=0$.

$y_{\text {lab }}=y_{\text {target }}+y_{\text {projectile }}=y_{\text {beam }}$ Hence mid-rapidity in fixed-target experiment is given by,

$$
y_{\text {mid }}^{L S}=y_{\text {beam }} / 2 \text {. }
$$

In collider experiments (center of mass system),

$y_{\text {projectile }}=-y_{\text {target }}=y_{C M S}=y_{\text {beam }} / 2$.

Hence, mid-rapidity in CMS system is given by

$$
y_{\text {mid }}^{C M S}=\left(y_{\text {projectile }}+y_{\text {target }}\right) / 2=0 .
$$

This is valid for a symmetric energy collider. The rapidity difference is given by $y_{\text {projectile }}-$ $y_{\text {target }}=2 y_{C M S}$ and this increases with energy for a collider as $y$ increases with energy.

\section{Light-cone variables and Rapidity}

Consider a particle having rapidity $y$ and the beam rapidity is $y_{b}$. The particle has forward light-cone variable $x_{+}$with respect to the beam particle

$$
\begin{aligned}
x_{+} & =\frac{p_{0}^{c}+p_{z}^{c}}{p_{0}^{b}+p_{z}^{b}} \\
& =\frac{m_{T}^{c}}{m^{b}} e^{y-y_{b}}
\end{aligned}
$$

where $m_{T}^{c}$ is the transverse mass of $c$. Note that the transverse momentum of the beam particle is zero. Hence,

$$
y=y_{b}+\ln x_{+}+\ln \left(\frac{m_{b}}{m_{T}^{c}}\right)
$$

Similarly, relative to the target particle $a$ with a target rapidity $y_{a}$, the backward light-cone variable of the detected particle $c$ is $x_{-} . x_{-}$is related to $y$ by

$$
x_{-}=\frac{m_{T}^{c}}{m^{b}} e^{y_{a}-y}
$$

and conversely,

$$
y=y_{a}-\ln x_{-}-\ln \left(\frac{m_{a}}{m_{T}^{c}}\right) .
$$

In general, the rapidity of a particle is related to its light-cone momenta by

$$
y=\frac{1}{2} \ln \left(\frac{p_{+}}{p_{-}}\right)
$$

Note that in situations where there is a frequent need to work with boosts along z-direction, it's better to use $\left(y, \mathbf{p}_{\mathbf{T}}\right)$ for a particle rather than using it's 3-momentum, because of the simple transformation rules for $y$ and $\mathbf{p}_{\mathbf{T}}$ under Lorentz boosts. 
8. The Maximum Accessible Rapidity in an Interaction

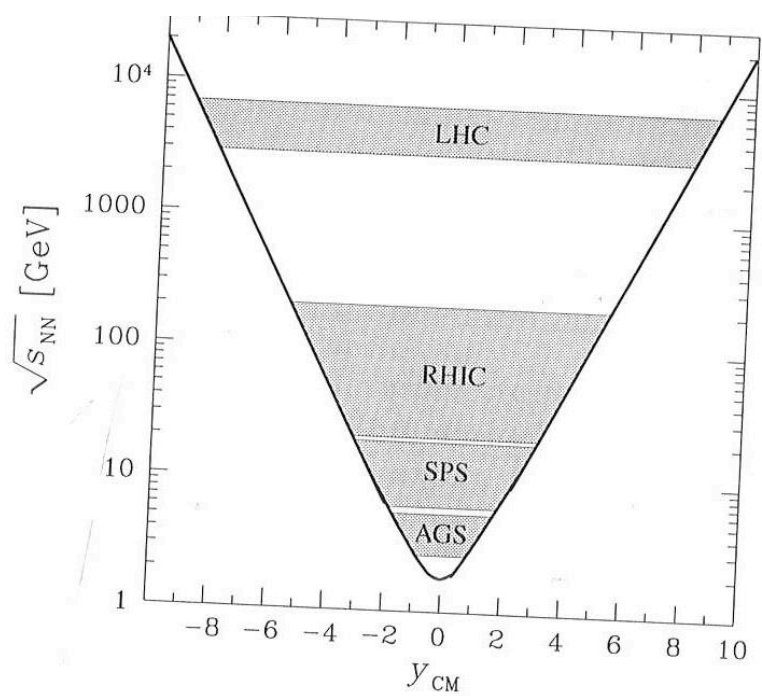

FIG. 8: $\sqrt{s_{N N}}$ (vertical axis) of various accelerators as a function of the projectile and target rapidities seen from the CM frame. Shaded areas: energy ranges accessible at various accelerators [3].

We know the variable rapidity " $y$ " of a particle is defined by Eqn. 76 :

$$
y=\frac{1}{2} \ln \left(\frac{E+p_{z}}{E-p_{z}}\right) .
$$

This corresponds to

$$
\tanh (y)=\frac{p_{z}}{E}
$$

where, $p_{z}$ is the longitudinal momentum along the direction of the incident particle, $E$ is the energy, both defined for a given particle. The accessible range of rapidities for a given interaction is determined by the available centre-of-mass energy and all participating particles' rest masses. One usually gives the limit for the incident particle, elastically scattered at zero angle:

$$
\begin{aligned}
|y|_{\text {max }} & =\ln \left[\left(E+p_{z}\right) / m\right] \\
& =\ln [\gamma+\gamma \beta] \\
& =\ln \left[\gamma+\sqrt{\gamma^{2}-1}\right] \\
& \simeq \cosh ^{-1} \gamma, \text { if } \gamma \gg 1
\end{aligned}
$$

with all variables referring to the through-going particle given in the desired frame of reference (e.g. in the centre of mass). A Lorentz boost $\beta$ along the direction of the incident particle adds a constant, $\ln [\gamma+\gamma \beta]$, to the rapidity. Rapidity differences, therefore, are invariant to a Lorentz boost. Statistical particle distributions are flat in $y$ for many physics production models. Note here that $\frac{\partial y}{\partial p_{z}}=1 / E$. 
Furthermore,

$$
\gamma=\frac{E_{\text {beam }}}{m_{p}}
$$

and for symmetric collision,

$$
E_{\text {beam }}=\frac{\sqrt{s_{N N}}}{2} \Rightarrow \gamma=\frac{\sqrt{s_{N N}}}{2 m_{p}}
$$

Hence,

$$
\begin{aligned}
y_{\max } & =\cosh ^{-1}\left[\frac{\sqrt{s_{N N}}}{2 m_{p}}\right] \\
& =\cosh ^{-1}\left[\frac{E_{l a b}}{A m_{p}}\right] \\
& \Rightarrow y_{\max }=y_{b}=\ln \left[\frac{\sqrt{s_{N N}}}{m_{p}}\right]
\end{aligned}
$$

Note that the maximum accessible rapidity is independent of the collision species for a symmetric collider and only depends on the center of mass energy.

\section{Examples:}

(a) For RHIC top energy, $\sqrt{s_{N N}}=200 \mathrm{GeV}, \gamma=E_{\text {beam }} / m_{p}=106.609$. Hence $\left.y\right|_{\max }=5.36$. For LHC, center of mass energy of $5.5 \mathrm{TeV}(\gamma=2931.768),\left.y\right|_{\max }=8.67$.

(b) $y_{\text {beam }}^{l a b}=5.8$ for $E_{l a b}=158 \mathrm{AGeV}$ and 4.4 for $E_{l a b}=40 \mathrm{AGeV}$.

\section{The Pseudorapidity Variable}

Let us assume that a particle is emitted at an angle $\theta$ relative to the beam axis. Then its rapidity can be written as

$y=\frac{1}{2} \ln \left(\frac{E+P_{L}}{E-P_{L}}\right)=\frac{1}{2} \ln \left[\frac{\sqrt{m^{2}+p^{2}}+p \cos \theta}{\sqrt{m^{2}+p^{2}}-p \cos \theta}\right]$. At very high energy, $p \gg m$ and hence

$$
\begin{aligned}
y & =\frac{1}{2} \ln \left[\frac{p+p \cos \theta}{p-p \cos \theta}\right] \\
& =-\ln \tan \theta / 2 \equiv \eta
\end{aligned}
$$

$\eta$ is called the pseudorapidity. Hence at very high energy,

$$
y \approx \eta=-\ln \tan \theta / 2 \text {. }
$$

In terms of the momentum, $\eta$ can be re-written as

$$
\eta=\frac{1}{2} \ln \left[\frac{|\mathbf{p}|+p_{z}}{|\mathbf{p}|-p_{z}}\right] .
$$

$\theta$ is the only quantity to be measured for the determination of pseudorapidity, independent of any particle identification mechanism. Pseudorapidity is defined for any value of mass, 


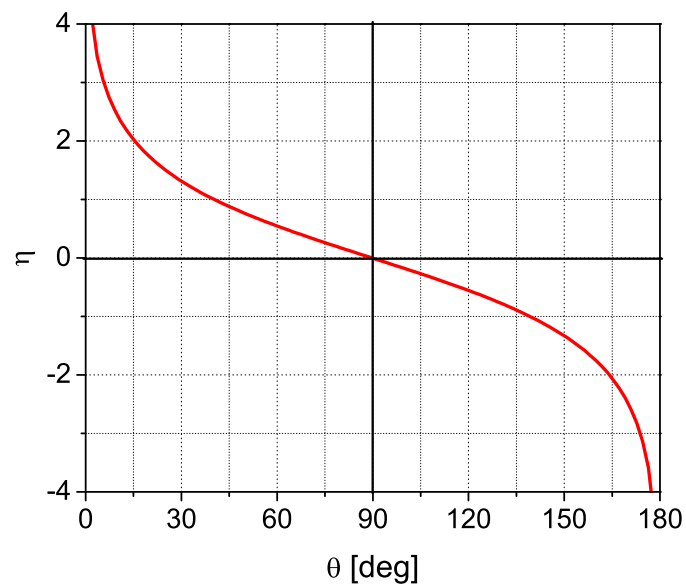

FIG. 9: A plot of pseudorapidity variable, $\eta$ as a function of the polar angle, $\theta$.

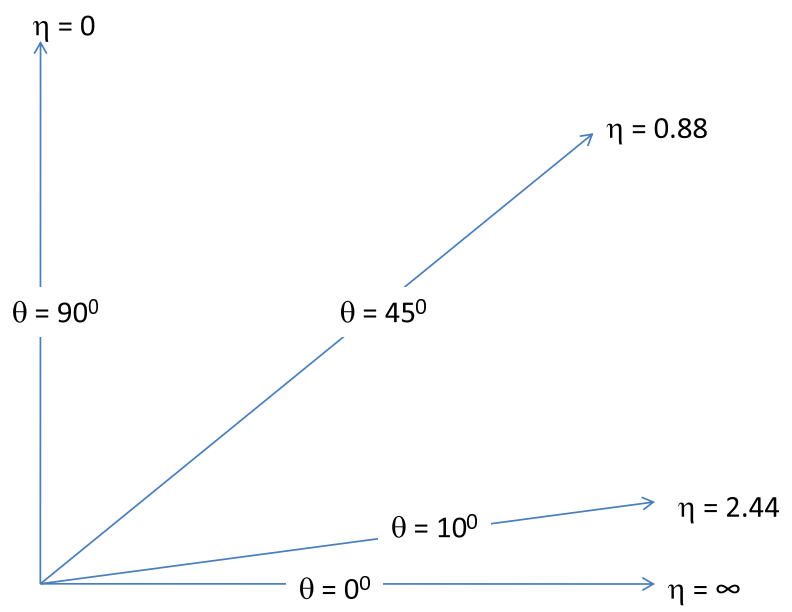

FIG. 10: As angle increases from zero, pseudorapidity decreases from infinity.

momentum and energy of the collision. This also could be measured with or without momentum information which needs a magnetic field. A plot of pseudorapidity as a function of the polar angle, $\theta$ is shown in Fig. 9. Table II shows the values of $\eta$ corresponding to the polar angle of emission a particle. One speaks of the "forward" direction in a collider experiment, which refers to regions of the detector that are close to the beam axis, at high $|\eta|$. The difference in the rapidity of two particles is independent of Lorentz boosts along the beam axis. Pseudorapidity is odd about $\theta=90$ degrees. In other words, $\eta(\theta)=-\eta(180-\theta)$.

TABLE II: Table of pseudorapidity, $\eta$ Vs the polar angle, $\theta$.

\begin{tabular}{|c|c|c|c|c|c|c|c|c|c|c|c|c|c|c|}
\hline$\theta$ & 0 & 5 & 10 & 20 & 30 & 45 & 60 & 80 & 90 & 100 & 110 & $\ldots$ & 175 & 180 \\
\hline$\eta$ & $\infty$ & 3.13 & 2.44 & 1.74 & 1.32 & 0.88 & 0.55 & 0.175 & 0 & -0.175 & -0.55 & $\ldots$ & -3.13 & $-\infty$ \\
\hline
\end{tabular}




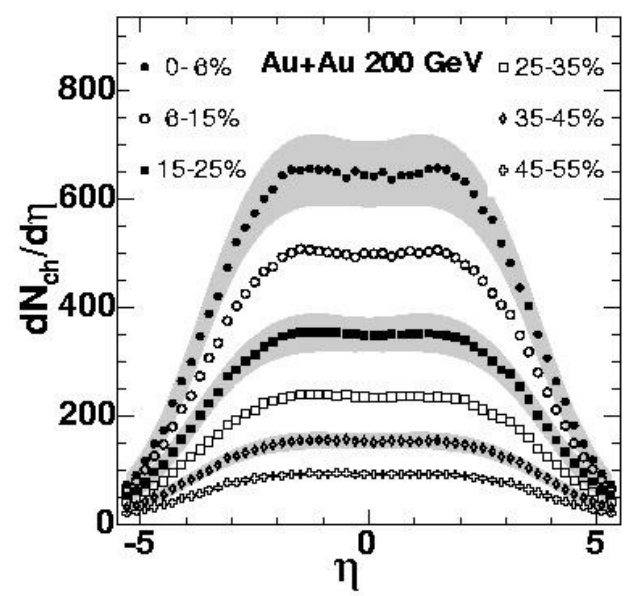

FIG. 11: The mid-rapidity $d N_{c h} / d \eta$ for $\mathrm{Au}+\mathrm{Au}$ collisions at $\sqrt{s_{N N}}=200 \mathrm{GeV}$ [6].

1. Change of variables from $\left(y, \mathbf{p}_{T}\right)$ to $\left(\eta, \mathbf{p}_{T}\right)$

By equation 119 ,

$$
\begin{array}{r}
e^{\eta}=\sqrt{\frac{|\mathbf{p}|+p_{z}}{|\mathbf{p}|-p_{z}}} \\
e^{-\eta}=\sqrt{\frac{|\mathbf{p}|-p_{z}}{|\mathbf{p}|+p_{z}}}
\end{array}
$$

Adding both of the equations, we get

$$
|\mathbf{p}|=p_{T} \cosh \eta
$$

$\mathbf{p}_{T}=\sqrt{|\mathbf{p}|^{2}-p_{z}^{2}}$. By subtracting the above equations, we get

$$
p_{z}=p_{T} \sinh \eta
$$

Using these equations in the definition of rapidity, we get

$$
y=\frac{1}{2} \ln \left[\frac{\sqrt{p_{T}^{2} \cosh ^{2} \eta+m^{2}}+p_{T} \sinh \eta}{\sqrt{p_{T}^{2} \cosh ^{2} \eta+m^{2}}-p_{T} \sinh \eta}\right]
$$

Similarly $\eta$ could be expressed in terms of $y$ as,

$$
\eta=\frac{1}{2} \ln \left[\frac{\sqrt{m_{T}^{2} \cosh ^{2} y-m^{2}}+m_{T} \sinh y}{\sqrt{m_{T}^{2} \cosh ^{2} y-m^{2}}-m_{T} \sinh y}\right]
$$

The distribution of particles as a function of rapidity is related to the distribution as a function of pseudorapidity by the formula

$$
\frac{d N}{d \eta d \mathbf{p}_{T}}=\sqrt{1-\frac{m^{2}}{m_{T}^{2} \cosh ^{2} y}} \frac{d N}{d y d \mathbf{p}_{T}} .
$$


In the region $y \gg 0$, the pseudorapidity distribution $(d N / d \eta)$ and the rapidity distribution $(d N / d y)$ which are essentially the $\mathbf{p}_{T}$-integrated values of $\frac{d N}{d \eta d \mathbf{p}_{T}}$ and $\frac{d N}{d y d \mathbf{p}_{T}}$ respectively, are approximately the same. In the region $y \approx 0$, there is a small "depression" in $d N / d \eta$ distribution compared to $d N / d y$ distribution due to the above transformation. At very high energies where $d N / d y$ has a mid-rapidity plateau, this transformation gives a small dip in $d N / d \eta$ around $\eta \approx 0$ (see Figure 11). However, for a massless particle like photon, the $\operatorname{dip}$ in $d N / d \eta$ is not expected (which is clear from the above equation). Independent of the frame of reference where $\eta$ is measured, the difference in the maximum magnitude of $d N / d \eta$ appears due to the above transformation. In the CMS, the maximum of the distribution is located at $y \approx \eta \approx 0$ and the $\eta$-distribution is suppressed by a factor $\sqrt{1-m^{2} /<m_{T}^{2}>}$ with reference to the rapidity distribution. In the laboratory frame, however the maximum is located around half of the beam rapidity $\eta \approx y_{b} / 2$ and the suppression factor is $\sqrt{1-m^{2} /<m_{T}^{2}>\cosh ^{2}\left(y_{b} / 2\right)}$, which is about unity. Given the fact that the shape of the rapidity distribution is independent of frame of reference, the peak value of the pseudorapidity distribution in the CMS frame is lower than its value in LS. This suppression factor at SPS energies is $\sim 0.8-0.9$.

It's to note here again that the conversion of rapidity to pseudorapidity phase space is associated with a Jacobian $J(y, \eta)$, which is given by the right hand side multiplier of Eqn. 126. This depends on the momentum distribution of the produced particles. In the limit of rest mass of the particles being much smaller than their momenta, $J(y, \eta)=1$. The value of the Jacobian is smaller at LHC energies, compared to that at RHIC energies, as the average transverse momentum of particles increases with beam energy. As measured by the PHENIX experiment, for central $\mathrm{Au}+\mathrm{Au}$ collisions at $\sqrt{s_{N N}}=200 \mathrm{GeV}, J(y, \eta)=1.25$. Whereas, the corresponding measurement of $J(y, \eta)=1.09$ for $\mathrm{Pb}+\mathrm{Pb}$ central collisions at $\sqrt{s_{N N}}=2.76 \mathrm{TeV}$ by the CMS experiment at LHC. Rewriting Eqn. 126 after taking an integration over $p_{T}$, one obtains:

$$
\frac{d N}{d \eta}=v(y) \frac{d N}{d y}
$$

where $v$ is the velocity of the particle. For a hadron of mass $m$ and momentum $p$, which emerges at an angle $90^{\circ}$ with respect to the beam direction, $y=\eta=0$, the above relationship becomes,

$$
\left.\frac{d N}{d \eta}\right|_{\eta=0}=\left.v \frac{d N}{d y}\right|_{y=0}
$$

As most of the particles are pions in the final state, with an average momentum of three times the pion mass, we have $v=0.95$. At mid-rapidity, the rapidity-pseudorapidity conversion hence involves with almost a constant factor. Hence the shape is not affected to a greater extent. However, when one considers the whole rapidity range, where the particle velocity in fact becomes a function of the rapidity, the shape of the pseudorapidity distribution (which is characterized by the width) is different from the rapidity distribution.

Usually, the rapidity spectra are parametrized by the sum of two Gaussian distributions positioned symmetrically with respect to mid-rapidity [7, 8],

$$
\frac{d N}{d y}=\frac{\langle N\rangle}{2 \sqrt{2 \pi \sigma^{2}}}\left\{\exp \left[-\frac{1}{2}\left(\frac{y-y_{0}}{\sigma}\right)^{2}\right]+\exp \left[-\frac{1}{2}\left(\frac{y+y_{0}}{\sigma}\right)^{2}\right]\right\}
$$

where $\langle N\rangle, \sigma$ and $y_{0}$ are fit parameters. $\sigma$ is the width of the rapidity distribution. 
Landau's energy dependent Gaussian rapidity distribution is given by [9, 10]

$$
\begin{aligned}
\frac{1}{\sigma_{\text {in }}} \frac{d \sigma}{d y} & =\frac{d N}{d y} \\
& =\frac{N}{(2 \pi L)^{1 / 2}} \exp .\left(-y^{2} / 2 L\right)
\end{aligned}
$$

with parameters

$$
\begin{aligned}
L & =\frac{1}{2} \ln \left(s / 4 m_{p}^{2}\right) \\
& =\ln \gamma=\ln \left(\sqrt{s_{N N}} / 2 m_{p}\right)
\end{aligned}
$$

where $s \equiv$ squared total center of mass energy. Comparing Eqns. 129 and 130, we get

$$
\sigma_{y}=\sqrt{\ln \left(\sqrt{s_{N N}} / 2 m_{p}\right)}
$$

$\sigma_{y}$ is the width of the rapidity distribution, with $m_{p}$ being the mass of proton. Interestingly, the width of the rapidity distribution is related to the longitudinal flow and velocity of sound in the medium and hence can probe the equation of state of the produced matter. However, this is affected by the final state rescattering, which could be understood through a $p_{T}$ dependent study of $\sigma_{y}$ to disentangle the initial hard scattering from the final state rescattering. With the assumption that the velocity of sound $c_{s}$ is independent of temperature, the rapidity density in the framework of Landau hydrodynamic model is given by [11, 12 ]

$$
\frac{d N}{d y}=K \frac{s_{N N}^{1 / 4}}{\sqrt{2 \pi \sigma_{y}^{2}}} \exp .\left(-\frac{y^{2}}{2 \sigma_{y}^{2}}\right)
$$

where

$$
\begin{array}{r}
\sigma_{y}^{2}=\frac{8}{3} \frac{c_{s}^{2}}{1-c_{s}^{4}} \ln \left(\sqrt{s_{N N}} / 2 m_{p}\right) \\
\Rightarrow \sigma_{y}^{2}=\frac{8}{3} \frac{c_{s}^{2}}{1-c_{s}^{4}} \ln \gamma
\end{array}
$$

and $K \equiv$ normalization factor. Inverting the above equation for $\sigma_{y}^{2}$, one can get [13]

$$
c_{s}^{2}=\frac{-4}{3} \frac{\ln \left(\sqrt{s_{N N}} / 2 m_{p}\right)}{\sigma_{y}^{2}}+\sqrt{\left[\frac{4}{3} \frac{\ln \left(\sqrt{s_{N N}} / 2 m_{p}\right)}{\sigma_{y}^{2}}\right]^{2}+1}
$$

For an ideal gas (Landau model prediction) the velocity of sound is, $c_{s}^{2}=1 / 3$.

However, for a gas of hadrons, $c_{s}^{2}=1 / 5$. This implies, the expansion of the system is slower compared to an ideal gas scenario. The equation of state is given by 

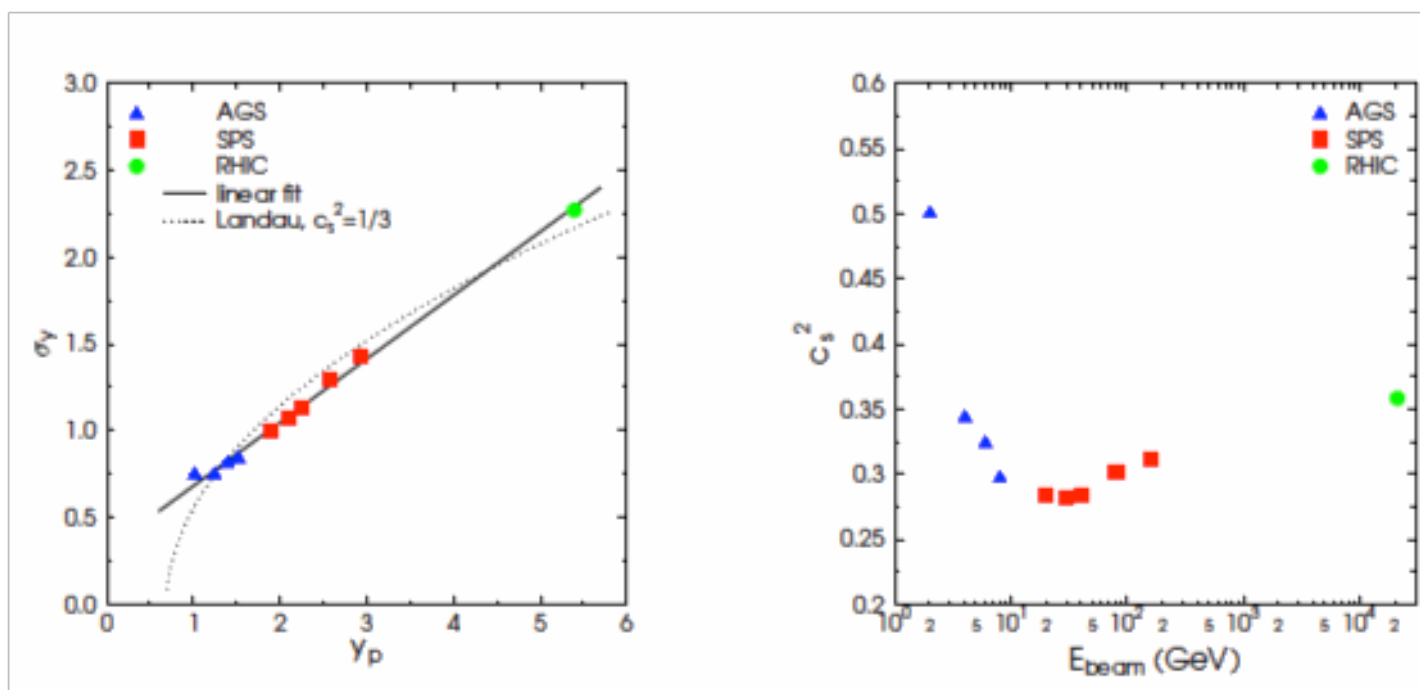

FIG. 12: Left: The width of rapidity distributions of $\pi^{-}$in central $\mathrm{Pb}+\mathrm{Pb}(\mathrm{Au}+\mathrm{Au})$ collisions as a function the beam rapidity. The dotted line indicates Landau model predictions with $c_{s}^{2}=1 / 3$, while the full line shows a linear fit to data points. Right: Speed of sound as a function of beam energy showing a softest point at $E_{\text {beam }}=30 \mathrm{~A} \mathrm{GeV} \mathrm{[13].}$

$$
\frac{\partial P}{\partial \epsilon}=c_{s}^{2}
$$

where, $P$ is the pressure and $\epsilon$ is the energy of the system under consideration. It should be noted here that when the expansion of the matter proceeds as longitudinal and superimposed transverse expansions, a rarefaction wave moves radially inwards with the velocity of sound. The velocity of sound in the medium formed in heavy-ion collisions, when studied as a function of collision (beam) energy, shows the softest point occurring around $E_{\text {beam }}=30$ AGeV. This could be a signature of deconfinement transition [13]. 


\begin{tabular}{l|l}
\hline \multicolumn{2}{c}{ Notation [14] } \\
\hline \multicolumn{1}{c}{ Coordinate Space } & \multicolumn{1}{c}{ Momentum Space } \\
\hline$(t, x, y, z) \rightarrow(\tau, r, \phi, \eta)$ & $\left(E, p_{x}, p_{y}, p_{z}\right) \rightarrow\left(m_{T}, \overrightarrow{p_{T}}, y\right)$ \\
$\tau=\sqrt{t^{2}-z^{2}}=$ proper time & $m_{T}=\sqrt{E^{2}-p_{z}^{2}}=$ transverse mass \\
$\eta=\tanh ^{-1} \frac{z}{t}=$ space-time rapidity & $y=\tanh ^{-1} \frac{p_{z}}{E}=\tanh ^{-1} v_{z}=$ rapidity \\
Conversely, & Conversely, \\
$t=\tau \cosh \eta, z=\tau \sinh \eta$ & $E=m_{T} \cosh y, p_{z}=m_{T} \sinh y$ \\
\hline
\end{tabular}

\section{N. The Invariant Yield}

The rapidity variable has the useful property that it transforms linearly under a Lorentz transformation so that the invariant differential single particle inclusive cross section becomes:

$$
\frac{E d^{3} \sigma}{d p^{3}}=\frac{E d^{3} \sigma}{p_{T} d p_{T} d p_{L} d \phi}=\frac{d^{3} \sigma}{p_{T} d p_{T} d y d \phi}
$$

where

$$
d y=\frac{d p_{L}}{E}
$$

First we proceed to show $\frac{d^{3} p}{E}$ is Lorentz invariant. The differential of Lorentz boost in longitudinal direction is given by

$$
d p_{z}^{*}=\gamma\left(d p_{z}-\beta d E\right)
$$

Taking the derivative of the equation $E^{2}=p^{2}+m^{2}$, we get

$$
E d E=p_{z} d p_{z}
$$

Using equations 139 and 140 we get

$$
\begin{aligned}
d p_{z}^{*} & =\gamma\left(d p_{z}-\beta \frac{p_{z} d p_{z}}{E}\right) \\
& =\frac{d p_{z}}{E} E^{*} .
\end{aligned}
$$

As $\mathbf{p}_{T}$ is Lorentz invariant, multiplying $\mathbf{p}_{T}$ on both the sides and re-arranging gives

$$
\frac{d^{3} p^{*}}{E^{*}}=\frac{d^{2} \mathbf{p}_{T} d p_{z}}{E}=\frac{d^{3} p}{E} .
$$

In terms of experimentally measurable quantities, $\frac{d^{3} p}{E}$ could be expressed as

$$
\begin{aligned}
\frac{d^{3} p}{E} & =d \mathbf{p}_{T} d y \\
& =p_{T} d p_{T} d \phi d y \\
& =m_{T} d m_{T} d \phi d y .
\end{aligned}
$$


The Lorentz invariant differential cross-section $\frac{E d^{3} \sigma}{d p^{3}}=\frac{E d^{3} N}{d p^{3}}$ is the invariant yield. In terms of experimentally measurable quantities this could be expressed as

$$
\begin{aligned}
\frac{E d^{3} \sigma}{d p^{3}} & =\frac{1}{m_{T}} \frac{d^{3} N}{d m_{T} d \phi d y} \\
& =\frac{1}{2 \pi m_{T}} \frac{d^{2} N}{d m_{T} d y} \\
& =\frac{1}{2 \pi p_{T}} \frac{d^{2} N}{d p_{T} d y} .
\end{aligned}
$$

To measure the invariant yields of identified particles equation 145 is used experimentally.

\section{O. Inclusive Production of Particles and the Feynman Scaling variable $x_{F}$}

A reaction of type

beam + target $\longrightarrow A+$ anything

where $A$ is known is called an "inclusive reaction". The cross-section for particle production could be written separately as functions of $\mathbf{p}_{T}$ and $p_{L}$ :

$$
\sigma=f\left(\mathbf{p}_{T}\right) g\left(p_{L}\right)
$$

This factorization is empirical and convenient because each of these factors has simple parametrizations which fit well to experimental data.

Similarly the differential cross-section could be expressed by

$$
\frac{d^{3} \sigma}{d p^{3}}=\frac{d^{2} \sigma}{\mathbf{p}_{T}^{2}} \frac{d \sigma}{d p_{L}}
$$

Define the variable

$$
\begin{gathered}
x_{F}=\frac{p_{L}^{*}}{p_{L}^{*}(\max )} \\
\Rightarrow x_{F}=\frac{2 p_{L}^{*}}{\sqrt{s}}
\end{gathered}
$$

$x_{F}$ is called the Feynman scaling variable: longitudinal component of the cross-section when measured in CMS of the collision, would scale i.e. would not depend on the energy $\sqrt{s}$. This is the fraction of maximum allowed longitudinal momentum $\left(-1 \leq x_{F} \leq 1\right)$ carried by the particle in the CMS. This is used in comparing the shapes of particle distributions at different collision energies near the projectile or target rapidity. Instead of $\frac{d \sigma}{d p_{L}^{*}}, \frac{d \sigma}{d x_{F}}$ is measured which wouldn't depend on energy of the reaction, $\sqrt{s}$. This Feynman's assumption is valid approximately. This variable is usually used to compare particle distribution at different collision energies.

The differential cross-section for the inclusive production of a particle is then written as

$$
\frac{d^{3} \sigma}{d x_{F} d^{2} \mathbf{p}_{T}}=F\left(s, x_{F}, \mathbf{p}_{T}\right)
$$


Feynman's assumption that at high energies the function $F\left(s, x_{F}, \mathbf{p}_{T}\right)$ becomes asymptotically independent of the energy means:

$$
\lim _{s \rightarrow \infty} F\left(s, x_{F}, \mathbf{p}_{T}\right)=F\left(x_{F}, \mathbf{p}_{T}\right)=f\left(\mathbf{p}_{T}\right) g\left(x_{F}\right)
$$

According to Feynman, the mean number of particles rises logarithmically in the asymptotic limit of large energies. This in fact applies to any kind of particles:

$$
<N>\propto \ln W \propto \ln \sqrt{s}
$$

where $W=\sqrt{s} / 2$, is the beam energy of a symmetric collider. However, these conclusions are based on phenomenological arguments about the exchange of quantum numbers between the colliding particles. It was assumed that the number of particles with a given mass and transverse momentum $\left(\mathbf{p}_{\mathrm{T}}\right)$ in a longitudinal interval $p_{z}$ depends on the energy $E=E\left(p_{Z}\right)$ as

$$
\frac{d N}{d p_{z}} \sim \frac{1}{E}
$$

This means the among all the produced particles, the probability of finding a particle of kind $i$ with transverse momentum $\mathbf{p}_{\mathrm{T}}$, mass $m$ and longitudinal momentum $p_{z}$ is of the form:

$$
f_{i}\left(\mathbf{p}_{\mathrm{T}}, x_{F}=p_{z} / W\right) \frac{d p_{z}}{E} d^{2} p_{T}
$$

with $E=\sqrt{m^{2}+p_{T}^{2}+p_{z}^{2}}$ being the total energy of the particle under discussion. The function $f_{i}\left(\mathbf{p}_{\mathrm{T}}, x_{F}\right)$ denotes the particle distribution. Let's derive Eqn. 151 to have a hand on experience.

Rewriting Eqn. 151 in the form of invariant cross section

$$
\frac{1}{\sigma} E \frac{d^{3} \sigma}{d p_{z} d^{2} \mathbf{p}_{T}}=f_{i}\left(\mathbf{p}_{\mathrm{T}}, x_{F}\right)
$$

$f_{i}$ factorizes approximately (found experimentally) and a normalization $g_{i}$ is chosen such that

$$
\begin{aligned}
\int f_{i}\left(\mathbf{p}_{\mathrm{T}}, x_{F}\right) d^{2} p_{T} & =f_{i}\left(x_{F}\right) \int g_{i}\left(\mathbf{p}_{T}\right) d^{2} p_{T} \\
& =f\left(x_{F}\right)
\end{aligned}
$$

Here

$$
\int g_{i}\left(\mathbf{p}_{T}\right) d^{2} p_{T}=1
$$

Now, integrating Eqn. 152 and applying Eqn. 153, we get

$$
\begin{aligned}
\int \frac{1}{\sigma} E \frac{d^{3} \sigma}{d p_{z} d^{2} \mathbf{p}_{T}} \frac{d^{3} p}{E} & =\langle N> \\
& =\int f_{i}\left(\mathbf{p}_{\mathrm{T}}, x_{F}\right) \frac{d^{3} p}{E} \\
& =\int f_{i}\left(x_{F}\right) \frac{d p_{z}}{\sqrt{W^{2} x_{F}^{2}+m_{T}^{2}}}
\end{aligned}
$$


On the left hand side we have used the definition of invariant cross section with the average particle multiplicity $\langle N\rangle$, and for $m_{\mathrm{T}}$ an effective average $p_{\mathrm{T}}$ is used with $x_{\mathrm{F}}=p_{\mathrm{z}} / W$. Hence

$$
<N>=\int_{-1}^{+1} f_{i}\left(x_{\mathrm{F}}\right) \frac{\mathrm{d} x_{F}}{\sqrt{x_{F}^{2}+\frac{m_{T}^{2}}{W^{2}}}}
$$

with $d x_{\mathrm{F}}=\frac{d p_{\mathrm{z}}}{W}$.

The integral is symmetric because $f_{i}\left(x_{\mathrm{F}}\right)$ is symmetric for collisions of identified particles. For asymmetric collision systems, the integration could be performed separately for negative and positive $x_{\mathrm{F}}$ and yields the same result.

$f_{i}\left(x_{\mathrm{F}}\right) \leq B$ is finite and bounded due to energy conservation. Feynman assumed that for $x_{\mathrm{F}}=0$ a finite limit is reached.

$$
\begin{aligned}
x_{\mathrm{F}} \rightarrow 0 \Rightarrow \frac{p_{z}}{W} & =\frac{p_{z}}{E_{l a b}} \rightarrow 0 \\
& \Rightarrow E_{l a b} \rightarrow \infty
\end{aligned}
$$

Hence

$$
\begin{aligned}
<N> & =2 \int_{0}^{1} f_{i}\left(x_{\mathrm{F}}\right) \frac{\mathrm{d} x_{F}}{\sqrt{x_{F}^{2}+\frac{m_{T}^{2}}{W^{2}}}} \leq 2 \int_{0}^{1} B \frac{\mathrm{d} x_{F}}{\sqrt{x_{F}^{2}+\frac{m_{T}^{2}}{W^{2}}}} \\
& =\left.2 B \ln \left[x_{F}+\sqrt{x_{F}^{2}+\frac{m_{T}^{2}}{W^{2}}}\right]\right|_{0} ^{1} \\
& =2 B \ln \left[1+\sqrt{1+\frac{m_{T}^{2}}{W^{2}}}\right]-2 B \ln \left(\frac{m_{T}}{W}\right)
\end{aligned}
$$

In the limit $W \rightarrow \infty$, the first term of the above equation could be shown to be constant and the second term is proportional to $\ln W$. hence, Feynman scaling tells that the average total multiplicity scales as:

$$
<N>\propto \ln W \propto \ln \sqrt{s}
$$

If one considers the maximum reachable rapidity in a collision to increase like $\ln \sqrt{s}$ $\left(y_{\max } \sim \ln \sqrt{s}\right)$ and in addition, particles are evenly distributed in rapidity, it follows that $\frac{d N}{d y}$ is independent of $\sqrt{s}$ :

$$
\frac{d N}{d y}=\text { Constant }
$$

Feynman's Assumption: $f_{i}\left(p_{\mathrm{T}}, x_{\mathrm{F}}\right)$ which denotes the particle distribution, becomes independent of $W$ at high energies. This assumption is known as Feynman Scaling and $f_{i}$ 
is called the scaling function or Feynman function. The variable

$$
x_{F}=p_{z} / W=2 p_{z} / \sqrt{s}
$$

is called Feynman-x.

Feynman- $x$ is the ratio of the longitudinal momentum of the particle to the total energy of the incident particle. Note:

1. $\frac{d N}{d y}=$ Constant $\Rightarrow$ Height of the rapidity distribution around mid-rapidity (the so-called plateau) is independent of collision energy, $\sqrt{s}$.

2. Equivalently, if Feynman scaling holds good, the pseudorapidity density of charged particles at mid-rapidity i.e. $\frac{d N}{d \eta}(\eta=0)$ is approximately constant.

Scaling properties of various distributions can also be studied in terms of the scaled rapidity:

$$
z=y^{*} / y_{\max }^{*}
$$

The two scaled variables: $x_{\mathrm{F}}$ and $z$ emphasize different kinematic regions: the detailed structure of the central part of the distribution (i.e. large emission angles) can be better seen using $x_{\mathrm{F}}$, while the far "wings" (i.e. small angles) using $z$.

\section{P. What is a $\mathbf{p}_{\mathrm{T}}$ or $m_{\mathrm{T}}$ Spectrum?}

$\mathbf{p}_{\mathrm{T}}$ spectrum: $\frac{1}{2 \pi p_{T}} \frac{d^{2} N}{d y d p_{T}} \operatorname{Vs} p_{\mathrm{T}}$

$m_{\mathrm{T}}$ spectrum: $\frac{1}{2 \pi m_{T}} \frac{d^{2} N}{d y d m_{T}} \operatorname{Vs} m_{\mathrm{T}}$ or $\left(m_{\mathrm{T}}-m_{0}\right)$

The invariant yield when plotted as a function of $\mathbf{p}_{\mathrm{T}}$ or $m_{\mathrm{T}}$ or $\left(m_{\mathrm{T}}-m_{0}\right)$ is called $\mathbf{p}_{\mathrm{T}}$ or $m_{\mathrm{T}}$ spectrum, respectively. Experimentally at kinetic freeze-out when the elastic collisions between the final state particles almost cease to happen (in other words the particle mean free path becomes higher than the system size: size of the produced fireball) then the $\mathbf{p}_{\mathrm{T}}$ or $m_{\mathrm{T}}$-spectrum is frozen, which carries the kinetic freeze-out properties of the system. Recall here that $p_{\mathrm{T}} d p_{\mathrm{T}}=m_{\mathrm{T}} d m_{\mathrm{T}}$.

The distribution of particles as a function of $\mathbf{p}_{T}$ is called $\mathbf{p}_{T}$-distribution. Mathematically,

$$
\frac{d N}{d \mathbf{p}_{T}}=\frac{d N}{2 \pi\left|\mathbf{p}_{T}\right| d\left|\mathbf{p}_{T}\right|}
$$

where $d N$ is the number of particles in a particular $\mathbf{p}_{T}$-bin. People usually plot $\frac{d N}{p_{T} d p_{T}}$ as a function of $p_{T}$ taking out the factor $1 / 2 \pi$ which is a constant. Here $p_{T}$ is a scalar quantity. The low- $p_{T}$ part of the $p_{T}$-spectrum is well described by an exponential function having thermal origin given by Eqn. (163). However, a QCD-inspired power-law function (given by Eqn. (164) seems to provide a better description of the high $p_{T}(\gtrsim 3 \mathrm{GeV} / \mathrm{c})$ region. To describe the whole range of the $p_{T}$-spectrum, one uses the Levy function given by Eqn.(165) 
which has an exponential part to describe low- $p_{T}$ and a power-law function to describe the hight- $p_{T}$ part which is dominated by hard scatterings (high momentum transfer at early times of the collision).

$$
\begin{gathered}
\frac{1}{2 \pi p_{T}} \frac{d^{2} N}{d y d p_{T}}=A e^{\frac{-m_{T}}{T}} \\
\frac{1}{2 \pi p_{T}} \frac{d^{2} N}{d y d p_{T}}=B\left(1+\frac{p_{T}}{p_{0}}\right)^{-n} \\
\frac{1}{2 \pi p_{T}} \frac{d^{2} N}{d y d p_{T}}=\frac{d N}{d y} \frac{(n-1)(n-2)}{2 \pi n C\left[n C+m_{0}(n-2)\right]} \times\left(1+\frac{\sqrt{p_{T}^{2}+m_{0}^{2}}-m_{0}}{n C}\right)^{-n}
\end{gathered}
$$

where $A, T, B, p_{0}, n, \frac{d N}{d y}, C$, and $m_{0}$ are fit parameters [15]. The inverse slope parameter of $p_{T}$-spectra is called the effective temperature $\left(T_{e f f}\right)$, which has a thermal contribution because of the random kinetic motion of the produced particles and a contribution from the collective motion of the particles. This will be described in details in the section of freeze-out properties and how to determine the chemical and kinetic freeze-out temperatures experimentally.

If we look deeper into Eqn. 163, we expect this for a 2-dimensional classical thermalized fluid at rest. We recall here that Boltzmann distribution $\propto \exp .(-\beta E)$, where $\beta \equiv 1 / T$ is the inverse temperature. We use a semi-logarithmic plot ( $y$-axis logarithmic scale) of $N \exp .\left(-\beta m_{T}\right)$ vs $\left(m_{T}-m_{0}\right)$ which happens to be a straight line with slope $-\beta$, from which the effective temperature is extracted. A semi-log plot of $N \exp .\left(-\beta m_{T}\right)$ vs $p_{T}$ is an approximate straight line if $p_{T} \gg m_{0}$.

The most important parameter is then the mean $p_{T}$ which carries the information of the effective temperature of the system. Experimentally, $\left\langle p_{T}\right\rangle$ is studied as a function of $\frac{d N_{c h}}{d \eta}$ which is the measure of the entropy density of the system. This is like studying the temperature as a function of entropy to see the signal of phase transition. The phase transition is of 1st order if a plateau is observed in the spectrum signalling the existence of latent heat of the system (like liquid-vapour phase transition). This was first proposed by L. Van Hove [16].

The average of any quantity $A$ following a particular probability distribution $f(A)$ can be written as

$$
\langle A\rangle=\frac{\int A f(A) d A}{\int f(A) d A} .
$$

Similarly,

$$
\begin{aligned}
\left\langle p_{T}\right\rangle & =\frac{\int_{0}^{\infty} p_{T}\left(\frac{d N}{d p_{T}}\right) d p_{T}}{\int_{0}^{\infty}\left(\frac{d N}{d p_{T}}\right) d p_{T}} \\
& =\frac{\int_{0}^{\infty} p_{T} d p_{T} \quad p_{T}\left(\frac{d N}{p_{T} d p_{T}}\right)}{\int_{0}^{\infty} p_{T} d p_{T}\left(\frac{d N}{p_{T} d p_{T}}\right)} \\
& =\frac{\int_{0}^{\infty} p_{T} d p_{T} p_{T} f\left(p_{T}\right)}{\int_{0}^{\infty} p_{T} d p_{T} f\left(p_{T}\right)}
\end{aligned}
$$


where $2 \pi p_{T} d p_{T}$ is the phase space factor and the $p_{T}$-distribution function is given by

$$
f\left(p_{T}\right)=\frac{d N}{d \mathbf{p}_{T}}=\frac{d N}{p_{T} d p_{T}} .
$$

Example Experimental data on $p_{T}$-spectra are sometimes fitted to the exponential Boltz-

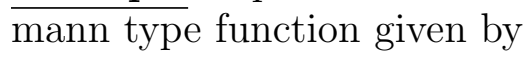

$$
f\left(p_{T}\right)=\frac{1}{p_{T}} \frac{d N}{d p_{T}} \simeq C e^{-m_{T} / T_{e f f}}
$$

The $\left\langle m_{T}\right\rangle$ could be obtained by

$$
\begin{aligned}
\left\langle m_{T}\right\rangle & =\frac{\int_{0}^{\infty} p_{T} d p_{T} m_{T} \exp \cdot\left(-m_{T} / T_{e f f}\right)}{\int_{0}^{\infty} p_{T} d p_{T} \exp \cdot\left(-m_{T} / T_{e f f}\right)} \\
& =\frac{2 T_{e f f}^{2}+2 m_{0} T_{e f f}+m_{0}^{2}}{m_{0}+T_{e f f}} \\
& \Rightarrow\left\langle m_{T}\right\rangle=T_{e f f}+m_{0}+\frac{\left(T_{e f f}\right)^{2}}{m_{0}+T_{e f f}}
\end{aligned}
$$

where $m_{0}$ is the rest mass of the particle. It can be seen from the above expression that for a massless particle

$$
\left\langle m_{T}\right\rangle=\left\langle p_{T}\right\rangle=2 T_{\text {eff }}
$$

This also satisfies the principle of equipartition of energy which is expected for a massless Boltzmann gas in equilibrium. However, in experiments the lower (higher) limit of $p_{T}$ is a finite quantity. In that case the integration will involve an incomplete gamma function.

\section{Q. How is the radial flow measured from $p_{\mathrm{T}}$-spectra?}

In central heavy-ion collisions, radial flow is supposed to play a vital role in the thermodynamic expansion of the produced fireball. Radial flow is related to the initial pressure produced just after the collision. This could be extracted from the analysis of the transverse momentum spectra. Assuming a thermalized non-relativistic plasma (for simplicity), particle velocity [14]

$$
\vec{v}=\vec{v}_{\text {flow }}+\vec{v}_{t h}
$$

where $\vec{v}_{\text {flow }} \equiv$ transverse velocity of the expanding fluid, which is independent of the particle species and is the collective component of $\vec{v}$.

$\vec{v}_{\text {flow }} \equiv$ thermal component of $\vec{v}$, which is generated due to random thermal motion of the quanta of the system.

Hence for a particle of mass $m_{0}$

$$
\begin{aligned}
\left\langle\frac{1}{2} m_{0} v^{2}\right\rangle & =\frac{1}{2} m_{0} v_{\text {flow }}^{2}+\left\langle\frac{1}{2} m_{0} v_{\text {th }}^{2}\right\rangle \\
& =\frac{1}{2} m_{0} v_{\text {flow }}^{2}+\frac{3}{2} k T,
\end{aligned}
$$


where $T$ is the temperature of the fluid. Hence the average kinetic energy (K.E) is given by

$$
\begin{aligned}
\langle K . E .\rangle & =\frac{1}{2} m_{0} v_{\text {flow }}^{2}+\frac{3}{2} k T \\
\Rightarrow \frac{3}{2} k T_{\text {eff }} & =\frac{1}{2} m_{0} v_{\text {flow }}^{2}+\frac{3}{2} k T_{\text {th }} \\
\Rightarrow T_{\text {eff }} & =T_{\text {th }}+\frac{1}{3} m_{0} v_{\text {flow }}^{2}(\text { taking } k=1)
\end{aligned}
$$

Because the final state particle are measured at freeze out (after they stream out to reach the detectors), the extracted values of $\vec{v}_{\text {flow }}$ and $T$ correspond to the instant of freeze out.

Taking $k=1$, in 2-dimension:

$$
T_{\text {eff }}=T_{\text {th }}+\frac{1}{2} m_{0} v_{\text {flow }}^{2} \text {, }
$$

and in 1-dimension:

$$
T_{\text {eff }}=T_{t h}+m_{0} v_{\text {flow }}^{2}
$$

Note that these formulae are used essentially when the spectra is well described by an exponential function (low- $p_{\mathrm{T}}$ regime). However, when one goes to high- $p_{\mathrm{T}}$ regime, the following formula could be used for extracting the radial flow from the $p_{\mathrm{T}}$ or $m_{\mathrm{T}}$-spectra.

$$
T_{\text {eff }}=T_{\text {th }} \sqrt{\frac{1+v_{\text {flow }}}{1-v_{\text {flow }}}}
$$

[1] Introduction to High Energy Physics, D. H. Perkins, Cambridge University Press, 4th edition (2000).

[2] Relativistic Kinematics, R. Hagedorn, W. A. Benjamin, Inc (1964).

[3] Hadrons and Quark-Gluon Plasma, J. Letessier and J. Rafelski, Cambridge (2005).

[4] Introduction to High-Energy Heavy-Ion Collisions, C-Y. Wong, World Scientific (1994).

[5] M. Kliemant, R. Sahoo, T. Schuster and R. Stock, Lect. Notes Phys. 785, 23 (2010), arXiv:0809.2482 [nucl-ex]].

[6] B.B. Back et al., (PHOBOS Collaboration), Nucl. Phys. A 75728 (2005).

[7] C. Alt et al. (NA49 Collaboraion), Phys. Rev. C 77, 024903 (2008).

[8] S.V. Afanasiev et al. (NA49 Collaboration), Phys. Rev. C 66, 054902 (2002).

[9] P. Carruthers and Minh Duong-van, Phys. Rev. D 8, 859 (1973). 
[10] C.Y. Wong, Phys. Rev. C 78, 054902 (2008).

[11] P. Carruthers, Annals N.Y. Acad.Sci 229, 91 (1974).

[12] E.V. Shuryak, Yad. Fiz 16, 395 (1972).

[13] H. Petersen and M. Bleicher, PoS CPOD2006 (2006) 025.

[14] R.S. Bhalerao, Lecture Notes in SERC School on Nuclear Physics, IoP, Bhubaneswar (2006)

[15] B.I. Abelev et al., (STAR Collaboration), Phys. Rev. C 75, 064901 (2007).

[16] L. van Hove, Phys. Lett. B 118, 138 (1982).

[17] J. Gosset et al., Phys. Rev. C 16, 629 (1977).

[18] J. Adams et al., (STAR Collaboration), Phys. Rev. C 70, 054907 (2004).

[19] F. Karsch, Prog. Part. Nucl. Phys. 62, 503 (2009). 